\title{
Al-free di-trioctahedral substitution in chlorite and a ferri-sudoite end-member
}

\author{
VINCENT TRINCAL ${ }^{1, *}$, AND PIERRE LANARI $^{2}$ \\ ${ }^{1}$ Laboratoire Chrono-Environnement, UMR CNRS 6249, University of Bourgogne Franche-Comté, 16 Route de Gray, \\ F-25000 Besançon, France \\ ${ }^{2}$ Institute of Geological Sciences, University of Bern, Baltzestrasse 1+3, CH-3012 Bern, Switzerland
}

(Received 01 November 2015; revised 30 April 2016; Guest editor: Atsuyuki Inoue)

\begin{abstract}
A compilation of $\mathrm{Fe}^{3+}$-bearing chlorite analyses is used: (1) to investigate the Alfree di-trioctahedral (AFDT) substitution $2 \mathrm{Fe}^{3+}+\square=3\left(\mathrm{Mg}, \mathrm{Fe}^{2+}\right.$ ) in chlorite; and (2) to estimate the composition of a ferri-sudoite end-member $\left(\mathrm{Si}_{3} \mathrm{Al}\right)\left[\left(\mathrm{Fe}^{2+}, \mathrm{Mg}\right)_{2} \mathrm{Fe}_{2}^{3+} \square \mathrm{Al}\right] \mathrm{O}_{10}(\mathrm{OH})_{8}$ within the chlorite solid-solution domain. According to our observations, up to two $\mathrm{Fe}^{3+}$ cations might be allocated in the M2-M3 chlorite sites by the substitution AFDT, which does not involve Al. These unexpected observations were made possible by the development of $\mu$ XANES techniques allowing in situ measurements of $X \mathrm{Fe}^{3+}\left(\mathrm{Fe}^{3+} /\left(\mathrm{Fe}^{2+}+\mathrm{Fe}^{3+}\right)\right)$ in heterogeneous chlorite. Although further studies are required to confirm the crystallographic position of $\mathrm{Fe}^{3+}$ and refine its ionic/ magnetic behaviour in chlorite, this development creates opportunities for developing new geothermometers.
\end{abstract}

KEYWORDS: chlorite, $\mu$ XANES, geothermometry, sudoite.

Chlorite is a ubiquitous phyllosilicate composed of tetrahedral and octahedral layers according to the $14.2 \AA$ 2:1:1 arrangement (e.g. Meunier, 2005). The tetrahedral sheet hosts tri- or tetravalent cations $\left(\mathrm{Si}^{4+}, \mathrm{Al}^{3+}\right.$ or rarely $\mathrm{Ti}^{4+}, \mathrm{Fe}^{3+}$ ), while the octahedral one is composed of di- or trivalent cations (usually $\mathrm{Fe}^{2+}, \mathrm{Mg}^{2+}, \mathrm{Mn}^{2+}, \mathrm{Al}^{3+}$ and $\mathrm{Fe}^{3+}$ ). The general formula of chlorite is: $R_{6-x-3 y}^{2+} R_{x+2 y}^{3+} \square_{y}$ $\left(\mathrm{Si}_{4-x} R_{x}^{3+}\right) \mathrm{O}_{10}(\mathrm{OH})_{8}$, in which $R^{2+}$ represents divalent cations, $R^{3+}$ trivalent cations and $\square$ octahedral vacancies (e.g. Wiewióra \& Weiss, 1990). According to Bailey (1988), the chlorite structure is formed of two types of tetrahedral crystallographic sites (denoted T1 and T2) and two octahedral sites (denoted M1 and M2). Two other octahedral sites (M3 and M4) are located in the brucite

\footnotetext{
*E-mail: vincenttrincal@gmail.com

${ }^{\dagger}$ This work was originally presented during the session

'The many faces of chlorite', part of the Euroclay 2015 conference held in July 2015 in Edinburgh, UK.

DOI: $10.1180 /$ claymin.2016.051.4.09
}

interlayer space. $\mathrm{Al}^{\mathrm{VI}}$ or trivalent $\mathrm{Fe}^{3+}$ cations are located preferentially in the M4 site, while the divalent cations occupy the other sites (Bailey, 1988). There are three categories of chlorite in diagenetic and low- to high-grade metamorphic rocks: tri-trioctahedral (sum of octahedral cations close to 6 a.p.f.u), di-trioctahedral (5 a.p.f.u) and di-dioctahedral (4 a.p.f.u). The main chemical substitutions occurring in chlorite are: Tschermak (TK) $\mathrm{Si}_{(\mathrm{IV})}^{++}+$ $\left(\mathrm{Fe}^{2+}, \mathrm{Mg}^{2+}\right)_{(\mathrm{VI})}=\mathrm{Al}_{(\mathrm{IV})}^{3+}+\mathrm{Al}_{(\mathrm{VI})}^{3+}$; ferromagnesian (FM) $\mathrm{Mg}_{(\mathrm{VI})}^{2+}=\mathrm{Fe}_{(\mathrm{VI})}^{2+}$ and di-trioctahedral (DT) $2 \mathrm{Al}_{(\mathrm{VI})}^{3+}+\square_{(\mathrm{VI})}$ $=3\left(\mathrm{Mg}^{2+}, \mathrm{Fe}^{2+}\right)_{\mathrm{VID}}$. Two filling models are used in the literature for the distribution of cations on the crystallographic sites. The first model referred to as 'disorderly', considers that there is no preferred configuration (e.g. Inoue et al., 2009). The second model, referred to as 'ordered' is constrained by specific rules for filling cations in the crystallographic sites such as preferred configuration and $\mathrm{Fe}-\mathrm{Mg}$ equipartition (e.g. Vidal et al., 2001, 2005, 2006; Lanari et al., 2014a). These authors assume, following Holland et al. (1998), $\mathrm{Al}_{\mathrm{IV}}$ in $\mathrm{T} 2$, vacancies in $\mathrm{M} 1, \mathrm{Al}_{\mathrm{VI}}$ 
TABLE 1. Structural formulae for classic chlorite end-members and the new ferri-sudoite end-member.

\begin{tabular}{llllll}
\hline & $\mathrm{T} 1(2)$ & $\mathrm{T} 2(2)$ & $\mathrm{M} 1(1)$ & $\mathrm{M} 2-\mathrm{M} 3(4)$ & $\mathrm{M}$ (1) \\
\hline Amesite-Mg/corundophilite & $\mathrm{Si}, \mathrm{Si}$ & $\mathrm{Al}, \mathrm{Al}$ & $\mathrm{Al}$ & $\mathrm{Mg}, \mathrm{Mg}, \mathrm{Mg}, \mathrm{Mg}$ & $\mathrm{Al}$ \\
Amesite-Fe & $\mathrm{Si}, \mathrm{Si}$ & $\mathrm{Al}, \mathrm{Al}$ & $\mathrm{Al}$ & $\mathrm{Fe}, \mathrm{Fe}, \mathrm{Fe}, \mathrm{Fe}$ & $\mathrm{Al}$ \\
Clinochlore & $\mathrm{Si}, \mathrm{Si}$ & $\mathrm{Si}, \mathrm{Al}$ & $\mathrm{Mg}$ & $\mathrm{Mg}, \mathrm{Mg}, \mathrm{Mg}, \mathrm{Mg}$ & $\mathrm{Al}$ \\
Chamosite/daphnite & $\mathrm{Si}, \mathrm{Si}$ & $\mathrm{Si}, \mathrm{Al}$ & $\mathrm{Fe}$ & $\mathrm{Fe}, \mathrm{Fe}, \mathrm{Fe}, \mathrm{Fe}$ & $\mathrm{Al}$ \\
Sudoite-Mg & $\mathrm{Si}, \mathrm{Si}$ & $\mathrm{Si}, \mathrm{Al}$ & $\square$ & $\mathrm{Mg}, \mathrm{Mg}, \mathrm{Al}, \mathrm{Al}$ & $\mathrm{Al}$ \\
Sudoite-Fe & $\mathrm{Si}, \mathrm{Si}$ & $\mathrm{Si}, \mathrm{Al}$ & $\square$ & $\mathrm{Fe}, \mathrm{Fe}, \mathrm{Al}, \mathrm{Al}$ & $\mathrm{Al}$ \\
Al-free chlorite & $\mathrm{Si}, \mathrm{Si}$ & $\mathrm{Si}, \mathrm{Si}$ & $\mathrm{Mg}$ & $\mathrm{Mg}, \mathrm{Mg}, \mathrm{Mg}, \mathrm{Mg}$ & $\mathrm{Mg}$ \\
Pyrophyllite-gibbsite & $\mathrm{Si}, \mathrm{Si}$ & $\mathrm{Si}, \mathrm{Si}$ & $\square$ & $\mathrm{Al}, \mathrm{Al}, \mathrm{Al}, \square$ & $\mathrm{Al}$ \\
Ferri-sudoite & $\mathrm{Si}, \mathrm{Si}$ & $\mathrm{Si}, \mathrm{Al}$ & $\square$ & $(\mathrm{Fe}, \mathrm{Mg})_{2}, \mathrm{Fe}^{3+}, \mathrm{Fe}^{3+}$ & $\mathrm{Al}$ \\
& & & & & \\
\hline
\end{tabular}

The numbers in parentheses refer to the quantity of atoms (per structural formula) distributed in tetrahedral $(\mathrm{T})$ or octahedral $(\mathrm{M})$ sites.

in M4 then M1 and then, eventually, in M2-M3, Fe and $\mathrm{Mg}$ in M2-M3 then in M1. Following the equipartition assumption, $X \mathrm{Mg}\left(\mathrm{Mg}^{2+} /\left(\mathrm{Mg}^{2+}+\mathrm{Fe}^{2+}\right)\right)$ is considered to be equal between the M1 and M2-M3 sites. Several chlorite end-members are classically defined (Table 1): Al-free chlorite $\left(\mathrm{Si}_{4}\right)\left[\mathrm{Mg}_{6}\right] \mathrm{O}_{10}(\mathrm{OH})_{8}$, amesite $\left(\mathrm{Si}_{2} \mathrm{Al}_{2}\right)$ $\left[\left(\mathrm{Fe}^{2+}, \mathrm{Mg}\right)_{4} \mathrm{Al}_{2}\right] \mathrm{O}_{10}(\mathrm{OH})_{8}$, clinochlore $\left(\mathrm{Si}_{3} \mathrm{Al}\right)$ $\left[\mathrm{Mg}_{5} \mathrm{Al}\right) \mathrm{O}_{10}(\mathrm{OH})_{8}$, corundophilite $\left(\mathrm{Si}_{2} \mathrm{Al}_{2}\right)\left[\mathrm{Mg}_{4} \mathrm{Al}_{2}\right]$ $\mathrm{O}_{10}(\mathrm{OH})_{8}$, daphnite $\left(\mathrm{Si}_{3} \mathrm{Al}\right)\left[\mathrm{Fe}_{5}^{2+} \mathrm{Al}\right] \mathrm{O}_{10}(\mathrm{OH})_{8}$, pyrophyllite-gibbsite $\left(\mathrm{Si}_{4}\right)\left[\mathrm{Al}_{4} \square_{2}\right] \mathrm{O}_{10}(\mathrm{OH})_{8}$ and sudoite $\left(\mathrm{Si}_{3} \mathrm{Al}\right)\left[\left(\mathrm{Fe}^{2+}, \mathrm{Mg}\right)_{2} \square \mathrm{Al}_{3}\right] \mathrm{O}_{10}(\mathrm{OH})_{8}$. These endmembers do not include any ferric iron component.

For 15 years, many authors have emphasized the importance of $\mathrm{Fe}^{3+}$ in chlorite, particularly in terms of its use as a geothermometer (Laird, 1988; Vidal et al., 2006; Inoue et al., 2009; Bourdelle et al., 2013; Lanari et al., 2014a). An additional substitution: $\mathrm{Al}-\mathrm{Fe}^{3+}$ that is supposed to occur on the M4 site was defined but without defining a ferric chlorite end-member (Vidal et al., 2006). In this model, the incorporation of $\mathrm{Fe}^{3+}$ changes the chlorite composition and the activity of the other end-members. This choice of the M4 site is based on the results of Smyth et al. (1997) suggesting that $\mathrm{Fe}^{3+}$ in chlorite shows a strong preference for the M4 site where it substitutes for $\mathrm{Al}^{3+}$.

Several methods are available to measure $\mathrm{Fe}^{3+}$ in chlorite: chemical titration (Tschermak, 1891; Orcel, 1927; Hallimond et al., 1939; Brindley, 1951), Mössbauer (Pal et al., 1993; Aja \& Dyar, 2000; Inoue et al., 2009), X-ray photoelectron spectroscopy (XPS) (Inoue et al., 2010) and micro-X-ray absorption near-edge spectroscopy ( $\mu$-XANES) (Wilke et al.,
2001; Vidal et al., 2006; Rigault, 2010; Trincal et al., 2015).

\section{CHLORITE DATA FROM THE LITERATURE}

A compilation of almost 200 published data of chlorite structural formulae containing $\mathrm{Fe}^{3+}$ is reported in Table 2. These chlorites come from a wide range of rocks from various localities. Some are of detrital origin; another experienced diagenetic to metamorphic conditions as indicated by the oscillatory zoned chlorites from the Pic-de-Port-Vieux outcrop (Fig. 1; Trincal et al., 2015). In addition, 202 data from three localities were incorporated in this review (Inoue et al., 2009). Most of the chlorites selected contain (in a.p.f.u, see Table 2): 2.5 to $3.5 \mathrm{Si}^{4+}, 2$ to $4 \mathrm{Al}^{3+}$ (Fig. 2), 1.5 to $5.5 R^{2+}\left(\mathrm{Mg}^{2+}, \mathrm{Mn}^{2+}, \mathrm{Fe}^{2+}\right)$ (Fig. 3) and $<1 \mathrm{Fe}^{3+}$ (Fig. 4). These analyses are distributed between classical end-members and apparently follow the classical DT and TK substitutions (Figs 2,3) or the $\mathrm{Al}-\mathrm{Fe}^{3+}$ substitution (Fig. 4).

In diagenetic to metamorphic rocks, the $X \mathrm{Fe}^{3+}$ content of chlorite depends on the temperature and the oxygen fugacity that is controlled by the buffering assemblage, i.e. the bulk-rock composition (Lanari et al. 2014a). This control is supported by data from Inoue et al. (2009), which show relationships between sampling localities and chlorite structural formulae and oxidation state (Figs 2-4). It is therefore critical to look at the variability of $X \mathrm{Fe}^{3+}$ recorded by chlorite in one rock sample, for a given bulk-rock composition. In such cases the observed variability of $X \mathrm{Fe}^{3+}$ is caused by temperature variations, 
TABLE 2. Compilation of structural formulae for $\mathrm{Fe}^{3+}$-bearing chlorites, calculated on the basis of $\mathrm{O}=14$.

\begin{tabular}{|c|c|c|c|c|c|c|c|c|c|c|c|c|c|c|c|c|c|c|c|c|c|}
\hline $\mathrm{N}^{\circ}$ & Reference & Method & $\mathrm{SiO}_{2}$ & $\mathrm{TiO}_{2}$ & $\mathrm{Al}_{2} \mathrm{O}_{3}$ & $\mathrm{Fe}_{2} \mathrm{O}_{3}$ & $\mathrm{FeO}$ & $\mathrm{MgO}$ & $\mathrm{MnO}$ & $\begin{array}{l}\mathrm{CaO}+\mathrm{Na}_{2} \mathrm{O}+ \\
\mathrm{K}_{2} \mathrm{O}\end{array}$ & Others & Total & $\mathrm{Si}$ & $\mathrm{Ti}$ & ${ }^{\mathrm{IV}} \mathrm{Al}$ & ${ }^{\mathrm{VI}} \mathrm{Al}$ & $\mathrm{Fe}^{3+}$ & $\mathrm{Fe}^{2+}$ & $\mathrm{Mg}$ & $\mathrm{Mn}$ & $\square$ \\
\hline 1 & Trincal et al. (2015) & XAN & 25.17 & 0.01 & 22.76 & & 20.98 & 17.27 & 0.09 & 0.05 & 0.05 & 86.37 & 2.58 & & 1.42 & 1.33 & 0.68 & 1.04 & 2.64 & & 0.30 \\
\hline 2 & Trincal et al. (2015) & XAN & 26.11 & 0.01 & 22.93 & & 18.43 & 19.64 & 0.09 & 0.04 & 0.05 & 87.29 & 2.63 & & 1.37 & 1.35 & 0.38 & 1.13 & 2.95 & & 0.18 \\
\hline 3 & Trincal et al. (2015) & XAN & 25.74 & 0.01 & 23.25 & & 20.43 & 17.86 & 0.09 & 0.04 & 0.05 & 87.47 & 2.60 & & 1.40 & 1.37 & 0.53 & 1.14 & 2.69 & & 0.25 \\
\hline 4 & Trincal et al. (2015) & XAN & 25.98 & 0.01 & 23.65 & & 18.39 & 19.09 & 0.09 & 0.04 & 0.05 & 87.30 & 2.62 & & 1.38 & 1.42 & 0.32 & 1.19 & 2.87 & & 0.18 \\
\hline 5 & Trincal et al. (2015) & XAN & 25.53 & 0.01 & 23.16 & & 21.94 & 16.41 & 0.08 & 0.04 & 0.04 & 87.23 & 2.59 & & 1.41 & 1.37 & 0.74 & 1.04 & 2.48 & & 0.35 \\
\hline 6 & Trincal et al. (2015) & $\mathrm{XAN}$ & 25.76 & 0.02 & 22.91 & & 21.92 & 16.36 & 0.10 & 0.05 & 0.05 & 87.17 & 2.62 & & 1.38 & 1.37 & 0.70 & 1.08 & 2.48 & & 0.35 \\
\hline 7 & Trincal et al. (2015) & XAN & 26.39 & 0.01 & 23.00 & & 17.59 & 19.83 & 0.11 & 0.05 & 0.05 & 87.02 & 2.65 & & 1.35 & 1.38 & 0.36 & 1.08 & 2.97 & & 0.20 \\
\hline 8 & Trincal et al. (2015) & XAN & 25.75 & 0.02 & 23.17 & & 20.47 & 17.44 & 0.11 & 0.05 & 0.05 & 87.04 & 2.62 & & 1.38 & 1.39 & 0.53 & 1.15 & 2.64 & & 0.27 \\
\hline 9 & Trincal et al. (2015) & XAN & 25.85 & 0.01 & 23.55 & & 18.70 & 18.83 & 0.11 & 0.05 & 0.05 & 87.15 & 2.61 & & 1.39 & 1.42 & 0.33 & 1.22 & 2.84 & & 0.18 \\
\hline 10 & Trincal et al. (2015) & XAN & 25.55 & 0.02 & 23.05 & & 21.48 & 16.58 & 0.11 & 0.05 & 0.05 & 86.87 & 2.60 & & 1.40 & 1.37 & 0.72 & 1.02 & 2.52 & & 0.35 \\
\hline 11 & Debret et al. (2015) & XAN & 33.76 & & 12.69 & & 4.17 & 33.65 & & & 1.59 & 86.14 & 3.23 & & 0.77 & 0.66 & 0.20 & 0.14 & 4.80 & & 0.04 \\
\hline 12 & Debret et al. (2015) & XAN & 33.73 & & 13.54 & & 3.35 & 33.92 & & & 1.40 & 87.00 & 3.19 & & 0.81 & 0.70 & 0.17 & 0.16 & 4.79 & & 0.03 \\
\hline 13 & Debret et al. (2015) & XAN & 33.47 & & 13.24 & & 3.26 & 33.66 & & & 1.76 & 85.57 & 3.21 & & 0.79 & 0.71 & 0.13 & 0.13 & 4.81 & & 0.02 \\
\hline 14 & Zhang et al. (2011) & CHE & & & & & & & & & & & 2.67 & 0.01 & 1.33 & 1.24 & 0.47 & 1.51 & 2.75 & 0.01 & 0.19 \\
\hline 15 & Inoue et al. (2010) & XPS & 29.98 & & 15.56 & 6.36 & 16.45 & 16.68 & 0.40 & 0.33 & & 85.75 & 3.13 & & 0.87 & 1.05 & 0.50 & 1.44 & 2.60 & 0.04 & 0.33 \\
\hline 16 & Inoue et al. (2010) & XPS & 28.08 & 0.07 & 18.34 & 3.80 & 13.82 & 19.08 & 1.12 & 0.31 & & 84.60 & 2.93 & 0.01 & 1.07 & 1.18 & 0.30 & 1.21 & 2.97 & 0.10 & 0.19 \\
\hline 17 & Inoue et al. (2010) & XPS & 27.34 & 0.03 & 18.58 & 4.51 & 16.42 & 17.75 & 0.35 & 0.27 & & 85.24 & 2.87 & 0.00 & 1.13 & 1.17 & 0.36 & 1.44 & 2.78 & 0.03 & 0.18 \\
\hline 18 & Inoue et al. (2010) & XPS & 29.98 & 0.04 & 19.05 & 3.91 & 11.26 & 20.30 & 0.60 & 0.29 & & 84.45 & 3.03 & 0.00 & 0.97 & 1.30 & 0.30 & 0.95 & 3.06 & 0.05 & 0.30 \\
\hline 19 & Inoue et al. (2010) & XPS & 24.26 & 0.01 & 17.64 & 5.56 & 33.82 & 1.37 & 3.34 & 0.11 & & 86.09 & 2.85 & 0.00 & 1.15 & 1.29 & 0.49 & 3.32 & 0.24 & 0.33 & 0.31 \\
\hline 20 & Inoue et al. (2010) & XPS & 27.17 & 0.05 & 18.77 & 3.76 & 19.37 & 13.71 & 3.21 & 0.24 & & 86.29 & 2.89 & 0.00 & 1.11 & 1.25 & 0.30 & 1.73 & 2.18 & 0.29 & 0.21 \\
\hline 21 & Inoue et al. (2010) & XPS & 22.86 & 0.03 & 17.55 & 6.40 & 30.55 & 1.92 & 2.15 & 0.13 & & 81.59 & 2.80 & 0.00 & 1.20 & 1.33 & 0.59 & 3.13 & 0.35 & 0.22 & 0.35 \\
\hline 22 & Rigault (2010) & XAN & & & & & & & & & & & 2.63 & 0.01 & 1.36 & 1.41 & 0.13 & 2.51 & 1.84 & 0.01 & 0.08 \\
\hline 23 & Rigault (2010) & XAN & & & & & & & & & & & 2.71 & & 1.29 & 1.60 & 0.14 & 2.58 & 1.40 & 0.05 & 0.22 \\
\hline 24 & Rigault (2010) & XAN & & & & & & & & & & & 2.92 & & 1.08 & 1.62 & 0.10 & 1.82 & 2.11 & 0.02 & 0.31 \\
\hline 25 & Rigault (2010) & XAN & & & & & & & & & & & 2.86 & & 1.14 & 1.44 & 0.34 & 0.34 & 3.54 & & 0.32 \\
\hline 26 & Rigault (2010) & XAN & & & & & & & & & & & 2.78 & 0.01 & 1.21 & 1.10 & 0.79 & 0.79 & 2.94 & 0.03 & 0.34 \\
\hline 27 & Rigault (2010) & XAN & & & & & & & & & & & 2.57 & 0.01 & 1.42 & 1.29 & 0.75 & 1.84 & 1.79 & 0.01 & 0.30 \\
\hline 28 & Rigault (2010) & XAN & & & & & & & & & & & 2.93 & & 1.07 & 1.53 & 0.18 & 0.39 & 3.57 & & 0.32 \\
\hline 29 & Singer et al. (2009) & EXA & & & & & & & & & & & 2.67 & 0.03 & 1.31 & 0.33 & 1.74 & 1.51 & 2.22 & 0.01 & 0.38 \\
\hline 30 & Vidal et al. (2006) & XAN & & & & & & & & & & & 2.76 & & 1.24 & 1.37 & 0.49 & 2.14 & 1.62 & & 0.31 \\
\hline 31 & Vidal et al. (2006) & XAN & & & & & & & & & & & 2.72 & & 1.28 & 1.32 & 0.43 & 2.11 & 1.85 & & 0.24 \\
\hline 32 & Vidal et al. (2006) & XAN & & & & & & & & & & & 2.71 & & 1.29 & 1.31 & 0.14 & 2.58 & 1.84 & & 0.07 \\
\hline
\end{tabular}




\begin{tabular}{|c|c|c|c|c|c|c|c|c|c|c|c|c|c|c|c|c|c|c|c|c|c|}
\hline $\mathrm{N}^{\circ}$ & Reference & Method & $\mathrm{SiO}_{2}$ & $\mathrm{TiO}_{2}$ & $\mathrm{Al}_{2} \mathrm{O}_{3}$ & $\mathrm{Fe}_{2} \mathrm{O}_{3}$ & $\mathrm{FeO}$ & $\mathrm{MgO}$ & $\mathrm{MnO}$ & $\begin{array}{l}\mathrm{CaO}+\mathrm{Na}_{2} \mathrm{O}+ \\
\mathrm{K}_{2} \mathrm{O}\end{array}$ & Others & Total & $\mathrm{Si}$ & $\mathrm{Ti}$ & ${ }^{\mathrm{IV}} \mathrm{Al}$ & ${ }^{\mathrm{VI}} \mathrm{Al}$ & $\mathrm{Fe}^{3+}$ & $\mathrm{Fe}^{2+}$ & $\mathrm{Mg}$ & $\mathrm{Mn}$ & $\square$ \\
\hline 33 & Vidal et al. (2006) & $\mathrm{XAN}$ & & & & & & & & & & & 2.69 & & 1.31 & 1.31 & 0.14 & 2.60 & 1.82 & & 0.07 \\
\hline 34 & Zazzi et al. (2006) & MÖS & 30.70 & 0.25 & 22.90 & 1.42 & & 31.10 & 0.01 & $<0.20$ & 0.01 & & 2.88 & & 1.12 & 1.41 & 0.01 & 0.10 & 4.35 & & 0.15 \\
\hline 35 & Zazzi et al. (2006) & MÖS & 33.60 & 0.11 & 13.70 & 5.95 & & 32.90 & 0.13 & $<0.74$ & 0.01 & & 3.24 & & 0.76 & 0.78 & 0.13 & 0.30 & 4.73 & & 0.07 \\
\hline 36 & Brandt et al. (2003) & CHE & 26.15 & & 21.13 & 5.93 & 17.20 & 17.73 & & & & 88.14 & 2.67 & & 1.33 & 1.21 & 0.46 & 1.47 & 2.70 & & 0.17 \\
\hline 37 & Brandt et al. (2003) & CHE & 26.83 & & 21.64 & 6.07 & 13.30 & 20.67 & & & & 88.51 & 2.67 & & 1.33 & 1.21 & 0.45 & 1.11 & 3.07 & & 0.17 \\
\hline 38 & Aja \& Dyar (2002) & MÖS & 29.64 & 0.04 & 23.93 & & 37.94 & 8.11 & 0.29 & 0.05 & 0.01 & & 2.82 & & 1.19 & 1.49 & 0.30 & 2.72 & 1.15 & 0.03 & 0.30 \\
\hline 39 & Aja \& Dyar (2002) & MÖS & 33.90 & 0.06 & 23.77 & & 8.33 & 33.80 & 0.04 & 0.10 & 0.01 & & 2.83 & & 1.17 & 1.17 & 0.07 & 0.50 & 4.20 & 0.01 & 0.03 \\
\hline 40 & Aja \& Dyar (2002) & MÖS & 26.73 & 1.92 & 20.21 & & 41.25 & 7.00 & 0.29 & 1.49 & 1.11 & & 2.63 & 0.14 & 1.23 & 1.11 & 0.28 & 3.08 & 1.03 & 0.03 & 0.08 \\
\hline 41 & Billaut et al. (2002) & MÖS & 37.02 & & 35.61 & 0.89 & & 11.07 & 0.07 & 1.20 & & & 3.23 & & 0.77 & 3.11 & 0.03 & 0.02 & 1.65 & & 1.19 \\
\hline 42 & Billaut et al. (2002) & MÖS & 36.70 & & 33.04 & 3.74 & & 11.04 & & 0.65 & & & 3.32 & & 0.68 & 2.93 & 0.15 & 0.13 & 1.58 & & 1.20 \\
\hline 43 & Billaut et al. (2002) & MÖS & 36.81 & & 30.58 & 7.25 & & 8.13 & 0.11 & 1.14 & & & 3.42 & & 0.58 & 2.87 & 0.33 & 0.24 & 1.24 & 0.01 & 1.31 \\
\hline 44 & Bertoldi et al. (2001) & MÖS & 22.46 & 0.06 & 19.29 & 4.70 & 37.20 & 2.87 & 0.07 & & & 86.65 & 2.62 & 0.01 & 1.38 & 1.28 & 0.41 & 3.63 & 0.50 & 0.01 & 0.16 \\
\hline 45 & Bertoldi et al. (2001) & MÖS & 30.38 & 0.10 & 18.70 & 0.79 & 6.29 & 29.81 & 0.05 & & & 86.12 & 2.94 & 0.01 & 1.06 & 1.08 & 0.06 & 0.51 & 4.30 & 0.00 & 0.04 \\
\hline 46 & Lougear et al. (2000) & MÖS & & & & & & & & & & & 2.68 & & 1.32 & 1.18 & 0.18 & 1.95 & 2.68 & & 0.02 \\
\hline 47 & Mitra \& Bidyananda (2001) & MÖS & 27.69 & 0.02 & 22.26 & & 12.26 & 24.56 & 0.17 & 0.01 & 0.08 & 87.05 & 2.73 & 0.00 & 1.27 & 1.32 & 0.37 & 0.64 & 3.61 & 0.01 & 0.22 \\
\hline 48 & Mitra \& Bidyananda (2001) & MÖS & 26.74 & 0.06 & 21.77 & & 11.72 & 24.36 & 0.17 & 0.01 & 0.17 & 85.00 & 2.70 & 0.01 & 1.29 & 1.31 & 0.37 & 0.62 & 3.67 & 0.02 & 0.19 \\
\hline 49 & Mitra \& Bidyananda (2001) & MÖS & 27.42 & 0.05 & 22.11 & & 12.28 & 24.72 & 0.15 & 0.01 & 0.16 & 85.67 & 2.72 & 0.00 & 1.28 & 1.30 & 0.38 & 0.64 & 3.65 & 0.01 & 0.20 \\
\hline 50 & Smyth et al. (1997) & MÖS & 31.68 & 0.05 & 17.13 & 1.34 & 1.62 & 34.76 & 0.08 & & 0.05 & & 2.99 & 0.00 & 1.01 & 0.89 & 0.10 & 0.13 & 4.88 & 0.01 & 0 \\
\hline 51 & Malmström et al. (1996) & MÖS & & & & & & & & & & & 3.50 & & 0.50 & 0.70 & 0.10 & 0.10 & 4.90 & & 0.15 \\
\hline 52 & May et al. (1995) & & & & & & & & & & & & 3.00 & & 1.00 & 0.90 & 0.10 & 0.10 & 4.90 & & 0 \\
\hline 53 & Gregori \& Mercader (1994) & $\mathrm{CHE}$ & & & & & & & & & & & 3.12 & & 0.88 & 1.69 & 0.64 & 1.03 & 2.17 & & 0.73 \\
\hline 54 & Gregori \& Mercader (1994) & $\mathrm{CHE}$ & & & & & & & & & & & 3.01 & & 0.99 & 0.49 & 0.29 & 0.50 & 4.20 & & 0 \\
\hline 55 & Gregori \& Mercader (1994) & $\mathrm{CHE}$ & & & & & & & & & & & 2.76 & & 1.24 & 2.01 & 0.12 & 0.70 & 3.30 & & 0.45 \\
\hline 56 & Pal et al. (1993) & MÖS & 27.69 & 0.02 & 22.26 & & 12.26 & 24.56 & 0.17 & 0.01 & 0.08 & 87.05 & 2.73 & 0.00 & 1.27 & 1.32 & 0.38 & 0.63 & 3.61 & 0.01 & 0.21 \\
\hline 57 & Pal et al. (1993) & MÖS & 26.74 & 0.06 & 21.77 & & 11.72 & 24.36 & 0.17 & 0.01 & 0.17 & 85.00 & 2.70 & 0.00 & 1.29 & 1.30 & 0.37 & 0.62 & 3.67 & 0.01 & 0.19 \\
\hline 58 & Pal et al. (1993) & MÖS & 27.42 & 0.05 & 22.11 & & 12.28 & 24.72 & 0.15 & 0.01 & 0.16 & 86.90 & 2.71 & 0.00 & 1.29 & 1.30 & 0.38 & 0.64 & 3.65 & 0.01 & 0.19 \\
\hline 59 & Anceau (1992) & $\mathrm{CHE}$ & 35.70 & & 33.77 & 2.81 & 0.35 & 13.26 & 0.04 & & 0.06 & 86.00 & 3.24 & & 0.76 & 2.85 & 0.19 & 0.03 & 1.79 & & 1.14 \\
\hline 60 & Kawano \& Tomita (1991) & & 39.25 & 0.48 & 32.94 & 2.98 & & 9.49 & & 0.26 & 0.02 & & 3.54 & 0.03 & 0.43 & 3.07 & 0.20 & & 1.28 & & 1.41 \\
\hline 61 & Chamberlain et al. (1989) & & 24.40 & & 21.14 & & 32.65 & 11.74 & 0.07 & & & & 2.59 & & 1.41 & 1.24 & 0.16 & 2.73 & 1.86 & 0.01 & 0 \\
\hline 62 & Joswig \& Fuess (1990) & & & & & & & & & & & & 2.85 & & 1.15 & 0.97 & 0.18 & 0.28 & 4.55 & & 0.00 \\
\hline 63 & Zheng \& Bailey (1989) & & & & & & & & & & & & 2.96 & & 1.04 & 0.54 & 0.23 & 0.09 & 4.73 & & 0 \\
\hline 64 & Ahn \& Buseck (1988) & & & & & & & & & & & & 2.83 & & 1.17 & 4.32 & 0.07 & & & & 1.61 \\
\hline 65 & DeGrave et al. (1987) & & & & & & & & & & & & 2.88 & & 1.12 & 1.32 & 0.23 & 2.07 & 2.16 & 0.05 & 0.22 \\
\hline 66 & DeGrave et al. (1987) & & & & & & & & & & & & 2.68 & & 1.32 & 1.06 & 0.14 & 1.44 & 3.06 & 0.07 & 0 \\
\hline 67 & DeGrave et al. (1987) & & & & & & & & & & & & 2.64 & & 1.36 & 0.85 & 0.32 & 1.82 & 3.10 & & 0 \\
\hline
\end{tabular}


68 DeGrave et al. (1987)

69 Rule \& Bailey (1987)

70 Ballet et al. (1985)

71 Ballet et al. (1985)

72 Ballet et al. (1985)

73 Ballet et al. (1985)

74 Lin and Bailey (1985)

75 MacKenzie et al. (1983)

76 MacKenzie et al. (1983)

77 Borggaard et al. (1982)

78 Kittrick (1982)

79 Kittrick (1982)

80 Kittrick (1982)

81 Kittrick (1982)

82 Kittrick (1982)

83 Kodama et al. (1982)

84 Kodama et al. (1982)

85 Kodama et al. (1982)

86 Kramm (1980)

87 Kramm (1980)

88 Kramm (1980)

89 Phillips et al. (1980)

90 Phillips et al. (1980)

91 Goodman \& Bain (1979)

92 Goodman \& Bain (1979)

93 Goodman \& Bain (1979)

94 Goodman \& Bain (1979)

95 Fransolet \& Bourguignon (1978)

96 Fransolet \& Bourguignon (1978)

97 Sudo \& Shimoda (1978)

98 Sudo \& Shimoda (1978)

99 Alysheva et al. (1977)

100 Alysheva et al. (1977)

101 Alysheva et al. (1977)

102 Ericsson et al. (1977)

103 Ericsson et al. (1977)

$\begin{array}{llllllll}32.90 & 0.06 & 35.40 & 2.69 & & 14.73 & 0.01 & 0.01 \\ 20.95 & & 35.21 & & 8.28 & 22.88 & & 0.58 \\ 21.42 & 0.26 & 34.06 & 0.63 & 0.95 & 28.66 & & 0.51\end{array}$

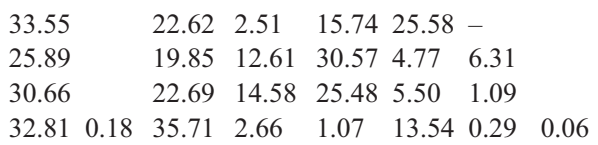

\begin{tabular}{|c|c|c|c|c|c|c|c|c|c|c|}
\hline & & 2.69 & & 1.31 & 1.55 & & 0.86 & 3.47 & & 0.12 \\
\hline & & 2.62 & & 1.38 & 1.28 & 0.14 & 1.16 & 2.96 & & 0.02 \\
\hline & & 2.64 & & 1.36 & 1.08 & 0.92 & 2.75 & 0.93 & & 0.32 \\
\hline & & 2.97 & 0.35 & 0.68 & 1.85 & 0.33 & 2.41 & 0.83 & & 0.75 \\
\hline & & 2.68 & & 1.32 & 1.18 & 0.18 & 1.95 & 2.69 & & 0.02 \\
\hline & & 3.00 & & 1.00 & 0.96 & 0.14 & 0.48 & 4.30 & & 0.05 \\
\hline \multirow[t]{15}{*}{0.07} & & 3.00 & & 1.00 & 2.81 & 0.18 & & 2.00 & & 1.00 \\
\hline & 88.90 & 2.01 & & 1.99 & 2.00 & & 0.66 & 3.28 & & 0.00 \\
\hline & 86.49 & 2.02 & 0.02 & 1.98 & 1.82 & 0.04 & 0.08 & 4.04 & & 0 \\
\hline & & 2.68 & & 1.32 & 1.17 & 0.18 & 1.95 & 2.69 & & 0.02 \\
\hline & & 2.97 & & 1.03 & 1.44 & 0.07 & 0.99 & 3.24 & & 0.24 \\
\hline & & 2.97 & & 1.03 & 1.44 & 0.07 & 0.99 & 3.24 & & 0.24 \\
\hline & & 2.99 & & 1.01 & 1.39 & 0.21 & 0.57 & 3.52 & & 0.30 \\
\hline & & 2.47 & & 1.53 & 1.60 & & 3.29 & 1.05 & & 0.04 \\
\hline & & 2.84 & & 1.16 & 1.75 & 0.12 & 2.61 & 1.16 & & 0.36 \\
\hline & & 2.91 & & 1.09 & 1.23 & 0.16 & 1.14 & 3.31 & & 0.15 \\
\hline & & 2.61 & & 1.39 & 0.96 & 0.95 & 2.57 & 0.72 & 0.54 & 0.26 \\
\hline & & 2.89 & & 1.11 & 1.42 & 1.04 & 2.01 & 0.77 & 0.09 & 0.67 \\
\hline & & 2.99 & 0.01 & 1.00 & 2.85 & 0.18 & 0.08 & 1.84 & 0.02 & 1.01 \\
\hline & & 3.03 & & 0.97 & 2.86 & 0.14 & 0.14 & 1.89 & 0.03 & 1.01 \\
\hline & & 3.04 & & 0.96 & 2.87 & 0.13 & 0.14 & 1.88 & 0.04 & 1.02 \\
\hline 3.40 & & 3.02 & & 0.98 & 0.72 & 0.04 & 0.04 & 4.95 & & 0 \\
\hline \multirow[t]{14}{*}{3.57} & & 2.99 & & 1.01 & 0.71 & 0.06 & 0.06 & 4.92 & & 0 \\
\hline & & 2.85 & 0.09 & 1.07 & 1.26 & 0.08 & 0.26 & 4.24 & & 0.14 \\
\hline & & 3.12 & & 0.89 & 0.60 & 0.18 & 0.32 & 4.95 & & 0 \\
\hline & & 2.81 & & 1.20 & 1.01 & 3.02 & 0.64 & 0.79 & 0.33 & 1.42 \\
\hline & & 3.12 & & 0.88 & 1.47 & 0.13 & 3.18 & 0.73 & 0.12 & 0.35 \\
\hline & & 3.01 & & 0.99 & 2.84 & 0.19 & 0.02 & 1.91 & 0.02 & 1.02 \\
\hline & & 2.99 & & 1.01 & 2.86 & 0.17 & & 1.94 & 0.02 & 1.01 \\
\hline & & 3.12 & & 0.88 & 3.29 & 0.03 & & 1.39 & & 1.16 \\
\hline & & 3.18 & 0.01 & 0.81 & 3.12 & 0.01 & 0.01 & 1.59 & & 1.12 \\
\hline & & 2.97 & & 1.03 & 2.90 & 0.18 & 0.17 & 1.58 & & 1.03 \\
\hline & & 3.24 & & 0.76 & 2.67 & 0.13 & & 2.14 & & 0.99 \\
\hline & & 3.04 & 0.03 & 0.93 & 2.43 & 0.03 & 0.04 & 2.50 & & 0.77 \\
\hline & & 2.95 & & 1.05 & 0.46 & 0.08 & 0.07 & 2.39 & & 0 \\
\hline & & 2.82 & & 1.19 & 0.55 & 0.53 & 1.59 & 2.60 & 0.04 & 0 \\
\hline
\end{tabular}

(continued) 
$\mathrm{CaO}+\mathrm{Na}_{2} \mathrm{O}+$

104 Malysheva et al. (1977)

105 Spanu et al. (1977)

106 Spanu et al. (1977)

107 Spanu et al. (1977)

108 Tsukuki \& Honda (1977)

109 Honda (1975)

110 Vrublevskaya et al. (1975)

111 Kimbara \& Nagata (1974)

112 Kimbara \& Sudo (1973)

113 Kimbara et al. (1973)

114 Post \& Plummer (1972)

115 Post \& Plummer (1972)

116 Post \& Plummer (1972)

117 Post \& Plummer (1972)

118 Aleksandrova et al. (1972)

119 deWaal (1970)

120 de Waal (1970)

121 Shimoda (1970)

122 Drits \& Lazarenko (1967)

123 Gomes (1967)

124 Weaver et al. (1967)

125 Frenzel \& Sehembra (1965)

126 Horikoshi (1965)

127 Hayashi \& Oinuma (1964)

128 Tsukahara (1964)

129 Tsukahara (1964)

130 Banno (1964)

131 Caillère et al. (1962)

132 Brydon et al. (1961)

133 Bilgrami \& Howie (1960)

134 Nakamura (1960)

135 Lapham (1958)

136 Shimane \& Sudo (1958)

137 Shirozu (1958)

138 Shirozu (1958)

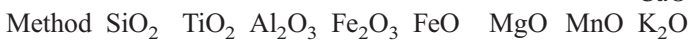

Others Total $\mathrm{Si} \quad \mathrm{Ti} \quad{ }^{\mathrm{IV}} \mathrm{Al}{ }^{\mathrm{VI}} \mathrm{Al} \mathrm{Fe}^{3+} \mathrm{Fe}^{2+} \mathrm{Mg} \quad \mathrm{Mn}$

$\begin{array}{llllllll}34.36 & & 38.84 & 0.49 & & 10.21 & & 0.85 \\ 38.26 & & 44.28 & 1.31 & 0.48 & & & \\ 33.10 & 0.30 & 37.43 & 0.87 & & 13.12 & & 0.44 \\ 29.45 & 0.20 & 20.14 & 4.08 & 18.69 & 14.93 & 0.36 & 1.59 \\ 30.84 & 0.12 & 14.15 & 2.59 & 18.42 & 20.62 & 0.10 & 0.84 \\ 25.80 & 18.30 & 4.10 & 21.30 & 20.30 & & \\ 27.90 & 25.20 & & 10.60 & 24.20 & & \\ 30.50 & 17.30 & 2.90 & 6.10 & 32.80 & & \\ 33.90 & 14.60 & 2.70 & 2.10 & 34.60 & & \\ & & & & & & & \\ 27.27 & 15.21 & 4.35 & 2.78 & 10.13 & 0.06 & 0.28 \\ 27.27 & 15.21 & 4.35 & 2.78 & 10.13 & 0.06 & 0.38 \\ 32.72 & 0.03 & 20.28 & 1.00 & & 24.47 & 0.06 & 2.22 \\ 31.17 & 37.49 & 1.04 & 1.95 & 14.25 & & 0.26\end{array}$

$\begin{array}{lllllllll}27.31 & 0.22 & 19.69 & 3.23 & 18.58 & 19.01 & 0.27 & 0.80\end{array}$ $\begin{array}{lllllll}35.63 & 34.87 & 5.01 & 0.43 & 8.63 & 0.05 & 1.83\end{array}$ $\begin{array}{lllllll}39.01 & 0.47 & 32.15 & 0.90 & 0.10 & 10.14 & 2.16\end{array}$ $\begin{array}{lllll}30.66 & 30.55 & 0.47 & 10.13 & 1.79\end{array}$ $\begin{array}{lllllllll}24.45 & 0.21 & 21.99 & 20.80 & 28.04 & 11.90 & 0.54 & 0.25\end{array}$

CHE

$\begin{array}{lllllllll}28.32 & 0.09 & 19.03 & 1.19 & 14.85 & 23.72 & 0.09 & 0.63\end{array}$ $\begin{array}{lllllllll}21.34 & 0.21 & 20.73 & 5.20 & 40.17 & 0.99 & 0.89 & 0.04\end{array}$ $\begin{array}{llllllll}36.43 & 12.24 & 0.94 & 6.87 & 30.94 & 0.11 & 0.33\end{array}$

$\begin{array}{lllllll}33.14 & 0.08 & 10.04 & 4.00 & 5.66 & 32.64 & 0.80\end{array}$

$\begin{array}{llllllll}29.73 & 17.95 & 0.68 & 10.05 & 21.84 & 8.24\end{array}$

$\begin{array}{llllllll}22.81 & 20.35 & 4.84 & 34.87 & 6.11 & 0.56 & 0.10\end{array}$ $\begin{array}{lllllll}1.46 & 0.90 & 1.13 & 2.60 & 1.10 & 0.28\end{array}$ $\begin{array}{lllllll}1.51 & 1.51 & 0.61 & 2.53 & 0.71 & 0.25 & 0.31\end{array}$

$\begin{array}{lllllllll}2.54 & 0.05 & 1.41 & 1.05 & 0.54 & 2.19 & 2.00 & 0.12 & 0.09\end{array}$ $\begin{array}{llllllll}2.55 & 1.45 & 1.37 & 0.33 & 1.50 & 2.65 & 0.03 & 0.13\end{array}$

$\begin{array}{lllllll}3.19 & 0.81 & 3.13 & 0.01 & 0.01 & 1.60 & 1.13\end{array}$ $\begin{array}{llllllll}85.75 & 3.12 & 0.88 & 3.29 & 0.03 & 1.38 & 1.16\end{array}$ $\begin{array}{lllllll}3.42 & 0.58 & 4.08 & 0.09 & 0.04 & 1.79\end{array}$ $\begin{array}{llllllll}85.26 & 3.01 & 0.02 & 0.97 & 3.05 & 0.06 & 1.78 & 1.04\end{array}$ $\begin{array}{llllllllll}89.34 & 2.97 & 0.02 & 1.02 & 1.36 & 0.31 & 1.58 & 2.24 & 0.03 & 0.27\end{array}$ $\begin{array}{llllllllll}87.68 & 3.15 & 0.01 & 0.84 & 0.87 & 0.20 & 1.58 & 3.14 & 0.01 & 0.09\end{array}$ $\begin{array}{lllllllll}89.80 & 2.64 & 1.36 & 0.85 & 0.32 & 1.82 & 3.10 & 0\end{array}$ $\begin{array}{llllllll}87.90 & 2.69 & 1.31 & 1.55 & 0.85 & 3.48 & 0.12\end{array}$ $\begin{array}{lllllllll}89.60 & 2.87 & 1.13 & 0.79 & 0.21 & 0.48 & 4.60 & 0\end{array}$ $\begin{array}{lllllllll}87.90 & 3.16 & 0.84 & 0.77 & 0.19 & 0.16 & 4.81 & 0.06\end{array}$ $\begin{array}{lllllll}3.14 & 0.86 & 4.10 & 0.04 & 0.01 & 0.08 & 1.64\end{array}$ $\begin{array}{lllllllllll}29.87 & 89.98 & 2.99 & & 1.01 & 0.95 & 0.36 & 0.25 & 1.65 & 0.01 & 0.15\end{array}$ $\begin{array}{llllllllll}29.87 & 2.98 & 1.02 & 0.95 & 0.36 & 0.25 & 1.65 & 0.01 & 0.14\end{array}$ $\begin{array}{lllllllll}80.78 & 3.24 & 0.00 & 0.76 & 1.61 & 0.07 & 3.61 & 0.01 & 0.44\end{array}$ $\begin{array}{llllllll}2.85 & 1.15 & 2.90 & 0.07 & 0.15 & 1.95 & 0.91\end{array}$ $\begin{array}{lllllllll}3.45 & 0.55 & 3.96 & 0.02 & 0.02 & 0.04 & 0.01 & 1.72\end{array}$ $\begin{array}{llllllll}2.66 & 1.34 & 1.74 & 0.02 & 3.68 & 0.42 & 0.21\end{array}$ $\begin{array}{lllllll}3.43 & 0.57 & 2.70 & 0.09 & 0.04 & 1.97 & 1.11\end{array}$ $\begin{array}{llllllllll}89.11 & 2.78 & 0.02 & 1.20 & 1.16 & 0.25 & 1.58 & 2.88 & 0.02 & 0.11\end{array}$ $\begin{array}{llllllll}86.45 & 3.26 & 0.74 & 3.02 & 0.34 & 0.04 & 1.17 & 1.31\end{array}$

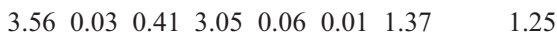
$\begin{array}{llllll}3.24 & 0.76 & 3.04 & 0.04 & 1.59 & 1.00\end{array}$ $\begin{array}{llllllllll}89.46 & 2.59 & 0.02 & 1.39 & 1.36 & 0.17 & 2.19 & 1.88 & 0.05 & 0.06\end{array}$ $\begin{array}{llllllll}2.70 & 1.30 & 4.30 & 0.03 & 0.02 & 1.52\end{array}$ $\begin{array}{llllllll}2.80 & 0.09 & 1.11 & 3.31 & 0.38 & 0.76 & 1.18\end{array}$ $\begin{array}{llllllllll}87.92 & 2.83 & 0.01 & 1.17 & 1.06 & 0.09 & 1.24 & 3.53 & 0.01 & 0\end{array}$ $\begin{array}{llllllllll}89.57 & 2.46 & 0.02 & 1.52 & 1.30 & 0.45 & 3.87 & 0.17 & 0.09 & 0.12\end{array}$ $\begin{array}{llllllllllll}0.63 & 88.49 & 3.43 & & 0.57 & 0.79 & 0.07 & 0.54 & 4.35 & 0.01 & 0.15\end{array}$ $\begin{array}{lllllllll}86.36 & 3.25 & 0.01 & 0.75 & 0.41 & 0.29 & 0.46 & 4.77 & 0\end{array}$ $\begin{array}{lllllllllll}88.49 & 2.98 & 1.02 & 1.09 & 0.05 & 0.84 & 3.26 & 0.70 & 0.06\end{array}$ $\begin{array}{llllllll}2.54 & 1.46 & 1.20 & 0.40 & 3.24 & 1.01 & 0.05 & 0.07\end{array}$ 
139 Shirozu (1958)

140 Shirozu (1958)

141 Shirozu (1958)

142 Shirozu (1958)

143 Battey (1956)

144 Frondel (1955)

145 Stone \& Weiss (1955)

146 Brindley \& Youell (1953)

147 Brindley \& Youell (1953)

148 Ginzburg (1953)

149 Mathias (1952)

150 Brindley (1951)

151 Brindley (1951)

152 Brindley (1951)

153 Brindley (1951)

154 Brindley (1951)

155 Bannister \& Whittard (1945)

156 Hutton \& Seelye (1945)

157 Hutton \& Seelye (1945)

158 Engelhardt (1942)

159 Engelhardt (1942)

160 Hödl (1942)

161 Hutton (1940)

162 Lazarenko (1940)

163 Lazarenko (1940)

164 Lazarenko (1940)

165 Hallimond et al. (1939)

166 Hallimond et al. (1939)

167 Hallimond et al. (1939)

168 Nockolds \& Richey (1939)

169 Holzner (1937)

170 Holzner (1937)

171 Hutton (1938)

172 Mélon (1938)

173 Tilley (1938)

174 Simpson (1937)

175 Simpson (1936)

176 Ross (1935) $\begin{array}{lllllll}29.71 & 19.75 & 0.29 & 6.59 & 30.79 & 0.30\end{array}$

$\begin{array}{lllllll}32.27 & 14.99 & 1.56 & 6.89 & 31.75 & 0.09\end{array}$

$\begin{array}{llllllll}27.11 & 0.35 & 17.42 & 2.91 & 30.98 & 9.75 & 0.21\end{array}$

$\begin{array}{lllllllll}29.94 & 0.16 & 13.77 & 10.46 & 20.96 & 10.71 & 0.18 & 1.34\end{array}$

$\begin{array}{lllllllll}28.73 & 0.41 & 19.16 & 1.97 & 10.99 & 26.37 & 0.15 & 0.10\end{array}$

$\begin{array}{llllll}22.47 & 21.82 & 0.22 & 37.24 & 2.57\end{array}$

$\begin{array}{llllll}23.48 & 22.45 & 43.37 & 0.45 & 2.60\end{array}$

$\begin{array}{lllll}33.40 & 47.47 & 0.71 & 0.20\end{array}$

0.54

$\begin{array}{llllllll}29.84 & 0.15 & 20.20 & 0.80 & 15.00 & 21.83 & 0.13 & 0.80\end{array}$

$\begin{array}{lllllllll}22.03 & 3.63 & 22.91 & 0.46 & 36.68 & 1.91 & 0.04 & 0.18\end{array}$

$\begin{array}{llllllll}21.40 & 0.89 & 25.40 & 0.25 & 37.60 & 2.04 & 0.05 & 0.13\end{array}$

$\begin{array}{lllllll}26.40 & 18.23 & 5.70 & 25.87 & 11.35 & 0.04 & 0.76\end{array}$

$\begin{array}{lllllllll}27.64 & 0.22 & 22.48 & 0.06 & 12.06 & 24.32 & 0.02 & 0.23\end{array}$

$\begin{array}{lllllll}31.87 & 0.17 & 14.51 & 1.86 & 3.57 & 32.76 & 0.06\end{array}$

$\begin{array}{lllll}20.82 & 17.64 & 8.70 & 37.96 & 4.15\end{array}$

$\begin{array}{llllll}26.65 & 16.14 & 6.69 & 34.43 & 4.47\end{array}$

$\begin{array}{lllll}22.47 & 23.57 & 4.01 & 29.27 & 9.81\end{array}$

$\begin{array}{lllllll}26.45 & 20.88 & 2.82 & 21.06 & 16.84 & 0.44 & 0.47\end{array}$

$\begin{array}{llllllll}24.35 & 0.04 & 20.21 & 2.13 & 36.27 & 5.57 & 0.48 & 0.10\end{array}$ $\begin{array}{llllll}24.90 & 15.60 & 7.20 & 35.00 & 4.60 & 0.40\end{array}$

$\begin{array}{lllllllll}25.07 & 0.12 & 19.78 & 3.50 & 35.80 & 1.11 & 0.50 & 2.15\end{array}$ $\begin{array}{lllllllll}25.09 & 0.05 & 18.94 & 9.60 & 25.66 & 9.80 & 0.02 & 0.49\end{array}$ $\begin{array}{lllllllll}22.18 & 0.04 & 20.04 & 7.35 & 35.23 & 3.79 & 0.02 & 0.49\end{array}$ $\begin{array}{lllllllll}26.69 & 0.30 & 19.57 & 3.49 & 21.80 & 16.23 & 0.30 & 0.17\end{array}$ $\begin{array}{lllllll}27.56 & 24.47 & 3.85 & 10.51 & 20.86 & 1.80\end{array}$

$\begin{array}{lllllllll}25.62 & 0.88 & 21.19 & 3.88 & 21.55 & 15.28 & 0.35 & 0.16\end{array}$ $\begin{array}{lllllllll}23.32 & 0.03 & 17.45 & 4.09 & 38.90 & 4.54 & 0.01 & 0.24\end{array}$ $\begin{array}{llllll}23.43 & 18.21 & 4.49 & 36.30 & 5.41\end{array}$

$\begin{array}{lllll}24.60 & 19.10 & 3.12 & 29.04 & 13.10\end{array}$

89.562 .63

$88.22 \quad 2.69$

87.432 .84

$87.55 \quad 3.10$ $\begin{array}{llllllllll}87.52 & 3.19 & 0.01 & 0.81 & 0.92 & 0.84 & 1.87 & 1.70 & 0.02 & 0.45\end{array}$ $\begin{array}{llllllllll}87.88 & 2.81 & 0.03 & 1.19 & 1.03 & 0.15 & 0.90 & 3.85 & 0.01 & 0\end{array}$ $\begin{array}{lllllllll}84.32 & 2.65 & 1.35 & 1.68 & 0.02 & 3.67 & 0.45 & 0.18\end{array}$ $\begin{array}{lllllllll}92.35 & 2.35 & 1.65 & 1.00 & 3.27 & 0.04 & 0.39 & 1.31\end{array}$

3.12

0.03

0.26

$0.93 \quad 4.22$

$0.05 \quad 0.03$

1.64

$\begin{array}{lllllllllll}7.93 & 0.01 & 1.07 & 1.27 & 0.06 & 1.23 & 3.19 & 0.01 & 0.12\end{array}$

$\begin{array}{lllllll}3.10 & 0.90 & 1.30 & 0.60 & 3.35 & 0.75 & 0.50\end{array}$

$\begin{array}{lllllllll}2.48 & 0.30 & 1.22 & 1.82 & 0.04 & 3.46 & 0.32 & 0.32\end{array}$

$\begin{array}{llllllllll}87.98 & 2.42 & 0.08 & 1.58 & 1.80 & 0.02 & 3.55 & 0.34 & 0.00 & 0.12\end{array}$ $\begin{array}{lllllllll}2.48 & 0.30 & 1.22 & 1.82 & 0.04 & 3.46 & 0.32 & 0.32\end{array}$ $\begin{array}{llllllllll}88.35 & 2.83 & & 1.17 & 1.13 & 0.46 & 2.32 & 1.81 & 0.00 & 0.18\end{array}$

$\begin{array}{lllllllllll}0.06 & 87.09 & 2.73 & 0.02 & 1.27 & 1.34 & 0.00 & 0.99 & 3.57 & 0.00 & 0.01\end{array}$

$\begin{array}{llllllllllll}1.38 & 86.18 & 3.07 & 0.01 & 0.93 & 0.72 & 0.14 & 0.29 & 4.71 & & 0\end{array}$ $\begin{array}{lllllllll}89.27 & 2.42 & 1.58 & 0.83 & 0.76 & 3.69 & 0.72 & 0.00\end{array}$ $\begin{array}{lllllllll}88.38 & 2.99 & 1.01 & 1.12 & 0.56 & 3.23 & 0.75 & 0.34\end{array}$

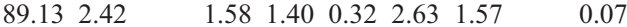
$\begin{array}{llllllllll}88.96 & 2.71 & 1.29 & 1.24 & 0.22 & 1.81 & 2.57 & 0.04 & 0.06\end{array}$ $\begin{array}{lllllll}3.09 & 0.91 & 3.92 & 0.05 & 0.21 & 1.44\end{array}$ $\begin{array}{llllll}3.06 & 0.94 & 3.88 & 0.08 & 0.23 & 1.40\end{array}$ $\begin{array}{lllllll}3.10 & 0.90 & 4.16 & 0.07 & 0.11 & 1.67\end{array}$ $\begin{array}{llllllll}2.80 & 1.20 & 0.86 & 0.60 & 3.56 & 0.78 & 0.04 & 0.13\end{array}$ $\begin{array}{lllllllllll}89.15 & 2.70 & 0.00 & 1.30 & 1.35 & 0.18 & 3.37 & 0.92 & 0.05 & 0.12\end{array}$ $\begin{array}{lllllllll}87.70 & 2.86 & 1.14 & 0.97 & 0.62 & 3.36 & 0.79 & 0.04 & 0.22\end{array}$ $\begin{array}{lllllllllll}88.03 & 2.85 & 0.01 & 1.15 & 1.50 & 0.30 & 3.40 & 0.19 & 0.05 & 0.24\end{array}$ $\begin{array}{lllllllllll}89.65 & 2.68 & 0.00 & 1.32 & 1.06 & 0.77 & 2.29 & 1.56 & 0.00 & 0.24\end{array}$ $\begin{array}{lllllllllll}90.60 & 2.51 & 0.00 & 1.49 & 1.18 & 0.63 & 3.33 & 0.64 & 0.00 & 0.15\end{array}$

$\begin{array}{lllllllllll}0.13 & 88.68 & 2.76 & 0.02 & 1.24 & 1.15 & 0.27 & 1.89 & 2.50 & 0.03 & 0.09\end{array}$ $\begin{array}{llllllllll}89.05 & 2.68 & 1.32 & 1.48 & 0.28 & 0.85 & 3.02 & 0.15 & 0.22\end{array}$ $\begin{array}{lllllllllll}88.91 & 2.64 & 0.07 & 1.36 & 1.22 & 0.30 & 1.86 & 2.35 & 0.03 & 0.08\end{array}$ $\begin{array}{lllllllllll}88.58 & 2.68 & 0.00 & 1.32 & 1.05 & 0.35 & 3.74 & 0.78 & 0.00 & 0.04\end{array}$ 87.842 .68

88.962 .64 $\begin{array}{lllll}1.32 & 1.13 & 0.39 & 3.47 & 0.92\end{array}$ $\begin{array}{lllll}1.36 & 1.06 & 0.25 & 2.61 & 2.10\end{array}$ 
TABLE 2. (contd.)

\begin{tabular}{|c|c|c|c|c|c|c|c|c|c|c|c|c|c|c|c|c|c|c|c|c|c|}
\hline $\mathrm{N}^{\circ}$ & Reference & Method & $\mathrm{SiO}_{2}$ & $\mathrm{TiO}_{2}$ & $\mathrm{Al}_{2} \mathrm{O}_{3}$ & $\mathrm{Fe}_{2} \mathrm{O}_{3}$ & $\mathrm{FeO}$ & $\mathrm{MgO}$ & $\mathrm{MnO}$ & $\begin{array}{l}\mathrm{CaO}+\mathrm{Na}_{2} \mathrm{O}+ \\
\mathrm{K}_{2} \mathrm{O}\end{array}$ & Others & Total & $\mathrm{Si}$ & $\mathrm{Ti}$ & ${ }^{\mathrm{IV}} \mathrm{Al}$ & ${ }^{\mathrm{VI}} \mathrm{Al}$ & $\mathrm{Fe}^{3+}$ & $\mathrm{Fe}^{2+}$ & $\mathrm{Mg}$ & $\mathrm{Mn}$ & $\square$ \\
\hline 177 & Jung (1931) & & 26.65 & & 16.14 & 6.69 & 34.43 & 4.47 & & & & 88.38 & 2.99 & & 1.01 & 1.12 & 0.56 & 3.23 & 0.75 & & 0.34 \\
\hline 178 & Dschang (1931) & & 30.30 & & 18.25 & 2.64 & 3.98 & 32.66 & & 0.03 & & 88.24 & 2.87 & & 1.13 & 0.90 & 0.19 & 0.32 & 4.61 & & 0 \\
\hline 180 & Orcel, 1927, p246 & & 22.27 & 0.08 & 21.40 & 0.67 & 43.01 & 2.35 & 0.05 & 0.15 & & 90.33 & 2.54 & 0.01 & 1.46 & 1.41 & 0.06 & 4.10 & 0.40 & 0.00 & 0.00 \\
\hline 181 & Orcel (1927) & & 26.50 & 0.03 & 20.85 & 1.90 & 18.73 & 19.85 & 0.52 & & & 88.38 & 2.69 & 0.00 & 1.31 & 1.19 & 0.15 & 1.59 & 3.01 & 0.04 & 0.01 \\
\hline 182 & Orcel (1927) & & 25.35 & & 22.06 & 18.97 & 8.36 & 15.06 & 0.08 & & & 89.88 & 2.52 & & 1.48 & 1.11 & 1.42 & 0.70 & 2.24 & 0.01 & 0.53 \\
\hline 183 & Orcel (1927) & & 27.12 & & 27.68 & 0.20 & 1.24 & 30.96 & 0.54 & & & 87.74 & 2.51 & & 1.49 & 1.54 & 0.01 & 0.10 & 4.28 & 0.04 & 0.03 \\
\hline 186 & Shannon \& Wherry (1922) & & 27.78 & & 24.30 & 1.43 & 0.35 & 32.71 & & & & 86.57 & 2.61 & & 1.39 & 1.29 & 0.10 & 0.03 & 4.58 & & 0.00 \\
\hline 187 & Poitevin \& Graham (1918) & & 24.40 & & 22.77 & 0.45 & & 32.70 & 0.09 & 0.40 & & 80.81 & 2.47 & & 1.53 & 1.18 & 0.03 & & 4.93 & 0.01 & 0 \\
\hline 188 & Dana (1915) & & 33.24 & & 11.07 & 2.26 & 25.11 & 16.51 & 0.41 & 1.36 & & 89.96 & 3.42 & & 0.58 & 0.77 & 0.18 & 2.16 & 2.53 & 0.04 & 0.16 \\
\hline 189 & Tschermak (1891) & & 23.62 & & 22.26 & & 38.97 & 1.09 & 0.98 & 1.67 & & 88.59 & 2.68 & & 1.32 & 1.67 & & 3.70 & 0.18 & 0.09 & 0.03 \\
\hline 190 & Tschermak (1891) & & 33.83 & & 12.95 & 2.25 & 3.02 & 34.94 & & & & 86.99 & 3.21 & & 0.79 & 0.65 & 0.16 & 0.24 & 4.94 & & 0.01 \\
\hline 191 & Hawes (1875) & & 33.46 & & 10.96 & 2.56 & 24.72 & 16.52 & 0.40 & 1.21 & & 89.83 & 3.44 & & 0.56 & 0.77 & 0.20 & 2.13 & 2.53 & 0.03 & 0.18 \\
\hline
\end{tabular}

Footnote: Speciation of Fe was obtained using chemical titration (CHE) or, for the past 20 years, using XANES (XAN), Mössbauer (MÖS) or XPS spectroscopies. Where no method is indicated, chemical titration is assumed. 


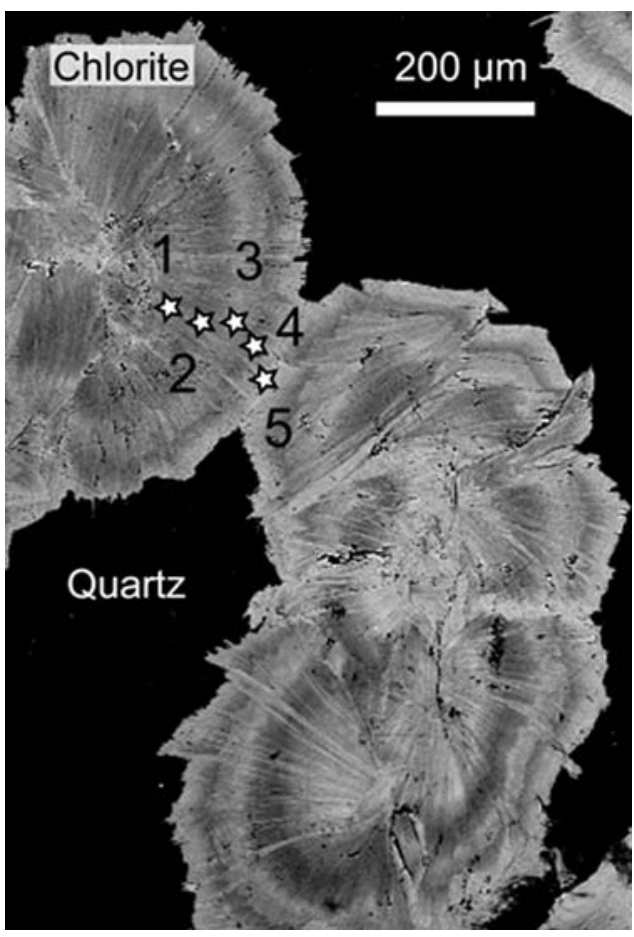

FIG. 1. Backscattered-electron image of an oscillatoryzoned chlorite showing Al-free di-trioctahedral substitution, from the Pic-de-Port-Vieux, Pyrenees, Spain. The numbers refer to the structural formulae reported in Table 2.

rather than by changes in the buffering assemblage (Lanari et al. 2014a; Trincal et al., 2015). Most of the spot analyses reported in the literature correspond to different geological environments (Table 2); it is thus very difficult to use them to evaluate the link between the $\mathrm{Fe}^{3+}$ behaviour and the evolution of a parameter such as pressure, temperature, $f_{\mathrm{O}_{2}}$, etc.

The choice of analytical method to estimate the $X \mathrm{Fe}^{3+}$ in chlorite is essential. Chlorite $\mathrm{Fe}^{3+}$ measurements by Mössbauer spectroscopy or titration are relatively common but provide few constraints due to poor spatial resolution. Indeed, recent investigations using high-resolution mapping (e.g. de Andrade et al., 2006; Muñoz et al., 2006) have demonstrated that chlorite is frequently zoned, recording strong compositional variability even at the crystal scale (e.g. Lanari et al., 2014b). Compositional zoning reflects time series of equilibrium conditions experienced by the rock. Thus in situ micrometric analyses are required to ensure a precise determination of the $\mathrm{Fe}^{3+} / \mathrm{Fe}_{\mathrm{Tot}}$ of the successive growth zones, which is impossible using Mössbauer spectroscopy. $\mu$-XANES $\mathrm{XFe}^{3+}$ spot analyses or mapping allow us to elucidate the heterogeneity of the $\mathrm{Fe}$ chemistry of chlorite which is critical to see the relations between formation temperature and crystal geometry (Vidal et al., 2006; Trincal et al., 2015).

\section{$\mu X A N E S$ DATA FROM ZONED CHLORITES}

Accurate in situ $\mu$ XANES and electron microprobe analyses performed on zoned chlorite from the Sambagawa metamorphic belt in Japan (Vidal et al., 2006) and on oscillatory-zoned chlorite from hydrothermal veins in the Pic-de-Port-Vieux, Pyrenees, Spain (fig. 1 from Trincal et al., 2015) show variations in the composition of the $\mathrm{Fe}^{3+}, \mathrm{Mg}$ and $\mathrm{Fe}^{2+}$ cation proportions, while that of $\mathrm{Si}$ and $\mathrm{Al}$ remain constant (Table 2, Fig. 2). This trend is observed independently in both samples, which have different bulk-rock compositions and experienced different metamorphic conditions. Indeed, chlorite from Japan comes from a blueschist-facies metapelite made of K-white mica, garnet, albite, quartz and graphite (Vidal et al., 2006), while the chlorite from the Pyrenees crystallizes in a vein of a greenschist-facies metapelite without garnet or graphite (Trincal et al., 2015). The absence of graphite is a good indicator of more oxidizing conditions. In both cases, $X \mathrm{Fe}^{3+}$ increases as temperature decreases, as suggested by numerous authors (e.g. Lanari et al., 2014a; Vidal et al., 2016). Furthermore, in both cases, there is no significant change in the $\mathrm{Al}$ content of the zoned chlorite. This result excludes the hypothesis of DT, TK or Al-Fe ${ }^{3+}$ substitutions being at the origin of the $X \mathrm{Fe}^{3+}$ variations.

\section{AL-FREE DI-TRIOCTAHEDRAL SUBSTITUTION}

Based on $\mu$ XANES analyses performed on zoned chlorite by Vidal et al. (2006) and Trincal et al. (2015), and in agreement with the literature data (Table 2), we demonstrate that a di-trioctahedral substitution implying $\mathrm{Fe}^{3+}$ can be used to explain the variations of $X \mathrm{Fe}^{3+}$ in natural chlorite:

$$
2 \mathrm{Fe}^{3+}+\square=3\left(\mathrm{Mg}^{2+}, \mathrm{Fe}^{2+}\right)
$$

As this substitution does not explicitly involve any Al cations, it is named $\mathrm{Al}$-free di-trioctahedral substitution (AFDT); it must not be confused with the Al-free chlorite end-member.

This substitution is similar to another couple suggested by Billault et al. (2002) and based on electron microprobe 


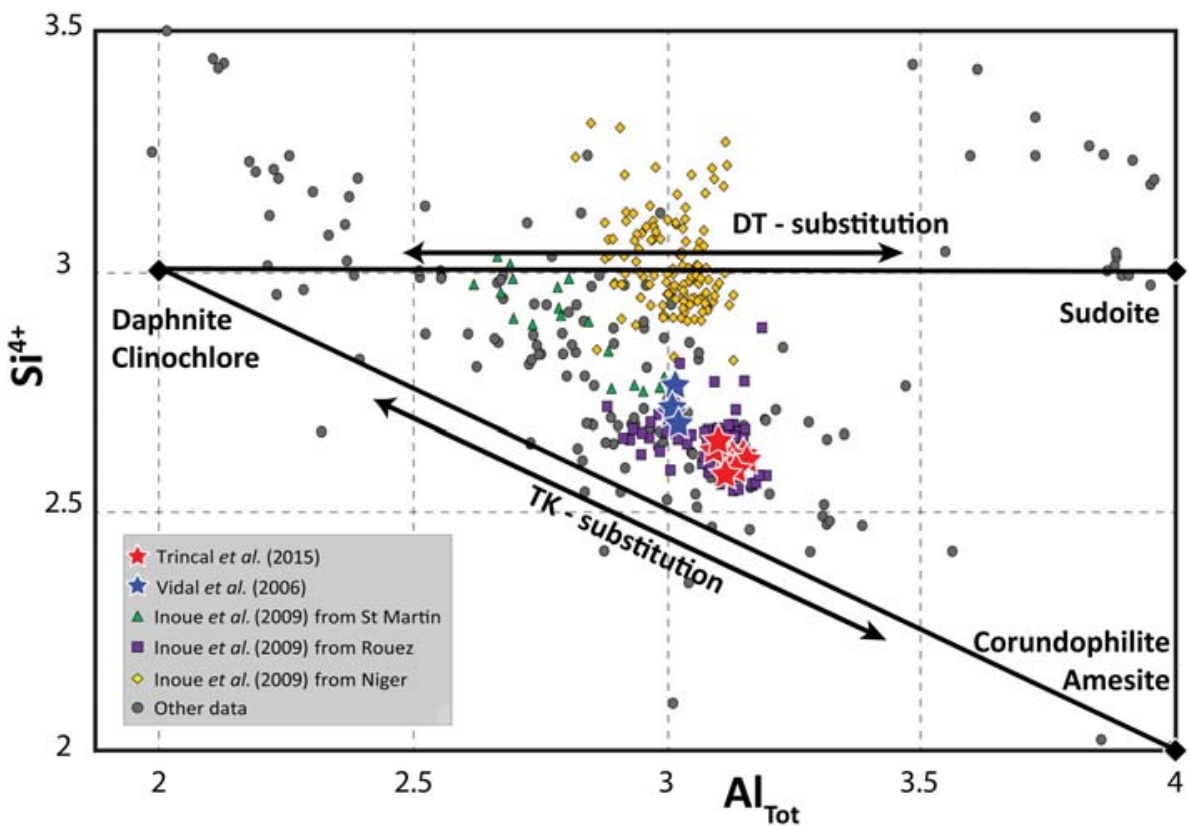

FIG. 2. Compilation of the $\mathrm{Fe}^{3+}$-bearing chlorite compositions. The $\mathrm{Si}$-Al plot is used to highlight the di-trioctahedral

(DT) and Tschermak (TK) substitutions. End-members and structural formulae are reported in Tables 1 and 2.

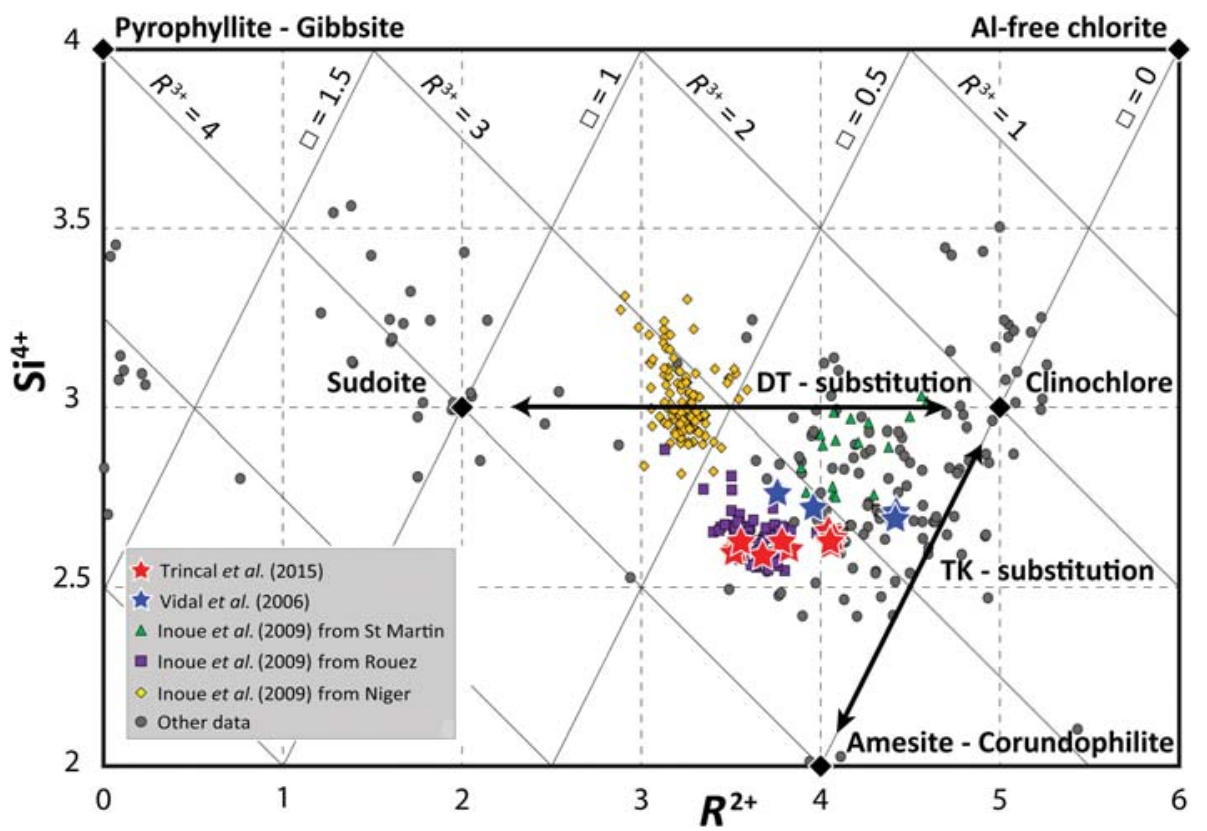

FIG. 3. Compilation of $\mathrm{Fe}^{3+}$-bearing chlorite compositions. The $\mathrm{Si}^{4+}-R^{2+}$ plot by Wiewióra \& Weiss (1990) is used to highlight the di-trioctahedral (DT) and the Al- $\mathrm{Fe}^{3+}$ substitutions. 


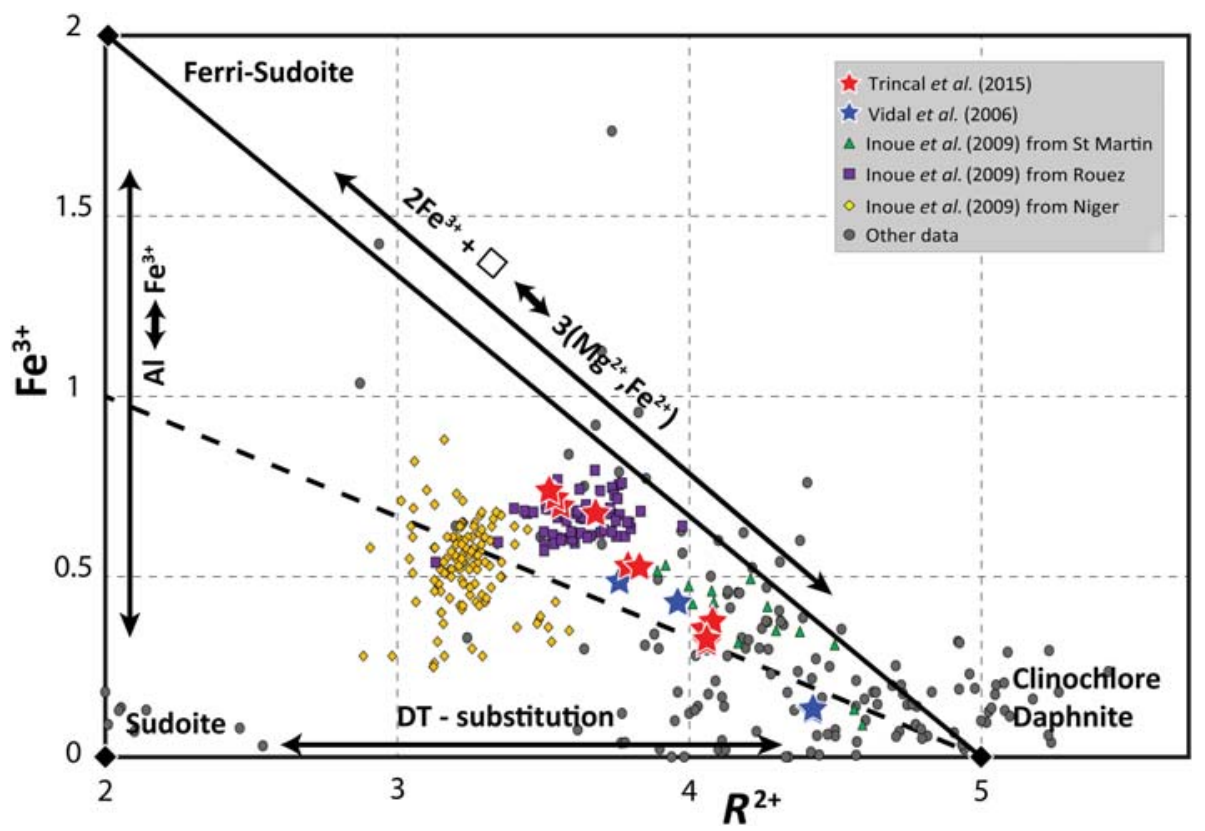

FIG. 4. Compilation of the $\mathrm{Fe}^{3+}$-bearing chlorite compositions. The $\mathrm{Fe}^{3+}-R^{2+}$ plot highlights the di-trioctahedral (DT), $\mathrm{Al}-\mathrm{Fe}^{3+}$ and AFDT substitutions.

and Mössbauer spectroscopic analyses on sudoite:

$$
\mathrm{Fe}^{3+}=\mathrm{Al}_{(\mathrm{VI})} \text { and } \mathrm{Fe}^{2+}+\mathrm{Fe}^{3+}+\square=3\left(\mathrm{Mg}^{2+}\right)
$$

However, chlorite analyses from their study display both $\mathrm{Al}$ and $X \mathrm{Mg}$ variations, which is not the case for the samples in the present study.

Chlorite compositions from Vidal et al. (2006) and Trincal et al. (2015) are plotted in an $\mathrm{Fe}^{3+} v$ s. $R^{2+}$ diagram (Fig. 4). In this diagram, data from zoned chlorite are distributed along a line parallel to the AFDT substitution vector. The AFDT substitution allows us to define a theoretical $\mathrm{Fe}^{3+}$-bearing chlorite end-member; namely ferri-sudoite $\left(\left(\mathrm{Si}_{3} \mathrm{Al}\right)\left[\left(\mathrm{Fe}^{2+}\right.\right.\right.$, $\left.\mathrm{Mg})_{2} \mathrm{Fe}_{2}^{3+} \square \mathrm{Al}\right) \mathrm{O}_{10}(\mathrm{OH})_{8}$ ) with two atoms of $\mathrm{Fe}^{3+}$ (Table 1). Ferri-sudoite can be separated into ferri-sudoite-Fe (( $\left.\left.\mathrm{Si}_{3} \mathrm{Al}\right)\left[\mathrm{Fe}_{2}^{2+} \mathrm{Fe}_{2}^{3+} \square \mathrm{Al}\right) \mathrm{O}_{10}(\mathrm{OH})_{8}\right)$ and ferri-sudoite- $\mathrm{Mg}\left(\left(\mathrm{Si}_{3} \mathrm{Al}\right)\left[\mathrm{Mg}_{2} \mathrm{Fe}_{2}^{3+} \square \mathrm{Al}\right) \mathrm{O}_{10}(\mathrm{OH})_{8}\right)$. As $\mathrm{Fe}^{3+}$ replaces $\mathrm{Mg}$ and $\mathrm{Fe}^{2+}$, it seems convenient to allocate up two atoms of $\mathrm{Fe}^{3+}$ to the M2-M3 sites (total multiplicity of 4) leaving $\mathrm{Al}$ in the M4 (Table 1).

According to several authors, the $\mathrm{Fe}^{3+}$ cation seems limited to 1 a.p.f.u and is generally allocated to the M4 site following the $\mathrm{Al}-\mathrm{Fe}^{3+}$ substitution. This last assertion is supported by Z-contrast images of highangle annular dark-field imaging-scanning transmission electron microscopy (HAADF-STEM) and other studies (e.g. Smyth et al., 1997; Inoue \& Kogure, 2016; Vidal et al., 2016). Among data collected in the literature, only six analyses show $\mathrm{Fe}^{3}$ ${ }^{+}$contents of $>1$ a.p.f.u (Orcel, 1927; Brindley \& Youell, 1953; Malysheva et al., 1977; Goodman \& Bain, 1979; Kodama et al., 1982; Singer et al., 2009 in Table 2). However, these analyses would actually match other phyllosilicates, suggesting contamination issues. Although AFDT substitution suggests that it is possible, no sudoite with $>1 \mathrm{Fe}^{3+}$ atom has been yet discovered; it offers interesting research opportunities.

\section{CONCLUSION}

The compilation of $\mathrm{Fe}^{3+}$-bearing chlorite analyses in this study, especially those acquired by $\mu$ XANES spectroscopy in zoned crystals, suggests the existence of a new chlorite substitution, the AFDT substitution that is characterized by $2 \mathrm{Fe}^{3+}+\square=3\left(\mathrm{Mg}^{2+}, \quad \mathrm{Fe}^{2+}\right)$ and implies a new chlorite end-member: ferri-sudoite $\left(\mathrm{Si}_{3} \mathrm{Al}\right)\left[\left(\mathrm{Fe}^{2+}, \mathrm{Mg}\right)_{2} \mathrm{Fe}_{2}^{3+} \square \mathrm{Al}\right) \mathrm{O}_{10}(\mathrm{OH})_{8}$ with 2 atoms of $\mathrm{Fe}^{3+}$. These results are not in agreement with previous studies which showed $\mathrm{Al}-\mathrm{Fe}^{3+}$ substitution implying only $1 \mathrm{Fe}^{3+}$. The absence of constraints on the $\mathrm{Fe}^{3+}$ crystallographic position together with the $\mathrm{Fe}^{3+}$ ionic properties in chlorite require further studies to confirm 
the existence of the AFDT substitution and of the ferrisudoite end-member, e.g. with accurate in situ $\mathrm{Fe}^{3+}$ measurements in synthetic or diagenetic chlorite formed in different oxidizing environments. This study opens the door for further research developments that would have strong implications, e.g. in chlorite geothermometry.

\section{ACKNOWLEDGEMENTS}

The authors thank Daniel Beaufort, Olivier Vidal and Martin Engi for fruitful discussions regarding $\mathrm{Fe}^{3+}$ in chlorite; Atsuyuki Inoue, Fernando Nieto-Garcia and another referee for the valuable advice about the manuscript; and Flavien Choulet for corrections to the language.

\section{REFERENCES}

Ahn J.H. \& Buseck P.R. (1988) Al-chlorite as a hydration reaction product of andalusite. Mineralogical Magazine, 52, 396-399.

Aja S.U. \& Dyar D.M. (2002) The stability of Fe-Mg chlorites in hydrothermal solutions-I. Results of experimental investigations. Applied Geochemistry, 17, 1219-1239.

Aleksandrova V.A., Drits V.A. \& Sokolova G.V. (1972) Structural features of dioctahedral one-packet chlorite. Soviet Physics-Crystallography, 17, 456-461.

Alysheva E.I., Rusinova O.V. \& Chekvaidze V.B. (1977) On sudoites from the polymetal deposits of Rydnyy Altai. Doklady Academii Nauk SSSR, 236, 722-724.

Anceau A. (1992) Sudoite in some Visean (lower Carboniferous) K-bentonites from Belgium. Clay Minerals, 27, 283-292.

Bailey S.W., editor (1988) Hydrous Phyllosilicates (exclusive of Micas). Reviews in Mineralogy, 19, Mineralogical Society of America, Washington, D.C.

Ballet O., Coey J.M.D. \& Burke K.J. (1985) Magnetic properties of sheet silicates; 2: 1: 1 layer minerals. Physics and Chemistry of Minerals, 12, 370-378.

Bannister F.A. \& Whittard W.F. (1945) A magnesian chamosite from the Wenlock Limestone of Wickwar, Gloucestershire. Mineralogical Magazine, 27, 99-111.

Banno S. (1964) Petrologic studies on Sanbagawa crystalline schists in the Bessi-Ino district, central Sikoku, Japan. Journal of Faculty Science, University of Tokyo, Section II, 15, 203-319.

Battey M.H. (1956) The petrogenesis of a spilitic rock series from New Zealand. Geological Magazine, 93, 89-110.

Bertoldi C., Benisek A., Cemic L. \& Dachs E. (2001) The heat capacity of two natural chlorite group minerals derived from differential scanning calorimetry. Physics and Chemistry of Minerals, 28, 332-336.

Bilgrami S.A. \& Howie R.A. (1960) The mineralogy and petrology of a rodingite dike, Hindubagh, Pakistan. American Mineralogist, 45, 791-801.
Billault V., Beaufort D., Patrier P. \& Petit S. (2002) Crystal chemistry of $\mathrm{Fe}$-sudoites from uranium deposits in the Athabasca basin (Saskatchewan, Canada). Clays and Clay Minerals, 50, 70-81.

Borggaard O.K., Lindgreen H.B. \& Morup S. (1982) Oxidation and reduction of structural iron in chlorite at $480^{\circ} \mathrm{C}$. Clays and Clay Minerals, 30, 353-364.

Bourdelle F., Benzerara K., Beyssac O., Cosmidis J., Neuville D.R., Brown G.E. Jr. \& Paineau E. (2013) Quantification of the ferric/ferrous iron ratio in silicates by scanning transmission X-ray microscopy at the Fe L2,3 edges. Contributions to Mineralogy and Petrology, 166, 423-434.

Brandt F., Bosbach D., Krawczyk-Bärsch E., Arnold T. \& Bernhard G. (2003) Chlorite dissolution in the acid pH range: a combined microscopic and macroscopic approach. Geochimica et Cosmochimica Acta, 67, 1451-1461.

Brindley G.W. (1951) The crystal structure of some chamosite minerals. Mineralogical Magazine, 29, 502-522.

Brindley G.W. \& Youell R.F. (1953) Ferrous chamosite and ferric chamosite. Mineralogical Magazine, 30, 57-70.

Brydon J.E., Clark J.S. \& Osborne V. (1961) Dioctahedral chlorite. The Canadian Mineralogist, 6, 595-609.

Caillère S., Hénin S. \& Pobeguin T. (1962) Présence d'un nouveau type de chlorite dans les 'bauxites' de SaintPaul-de-Fenouillet (Pyrénées orientales). Compte Rendu de l'Adadémie des Sciences, 254, 1657-1658.

Chamberlain S.C., Robinson G.W. \& Richards R.P. (1989) Mineralogy of the Alpine veins near Sherbrooke, Quebec. Mineralogical Record, 20, 209-20.

Chernykh V.V. (1926) Physicochemische Untersuchung der Serpentine und Chlorite. Mémoire de la Société Russe de Minéralogie Série 2, 55, 183-194.

Dana E.S. (1915) Descriptive Mineralogy. John Wiley \& Sons, New York, 659 pp.

De Andrade V., Vidal O., Lewin E., O'Brien P. \& Agard P. (2006) Quantification of electron microprobe compositional maps of rock thin sections: an optimized method and examples RID C-2856-2009. Journal of Metamorphic Geology, 24, 655-668.

Debret B., Bolfan-Casanova N., Padrón-Navarta J.A., Martin-Hernandez F., Andreani M., Garrido C.J., Sánchez-Vizcaíno V.L., Gómez-Pugnaire M.T., Muñoz M. \& Trcera N. (2015) Redox state of iron during high-pressure serpentinite dehydration. Contributions to Mineralogy and Petrology, 169, 1-18.

De Grave E., Vandenbruwaene J. \& Bockstael M.V. (1987) ${ }^{57} \mathrm{Fe}$ Mössbauer spectroscopic analysis of chlorite. Physics and Chemistry of Minerals, 15, 173-180.

De Waal S.A. (1970) Nickel minerals from Barberton, South Africa: III. Willemseite, a nickel-rich talc. American Mineralogist, 55, 31-42.

Drits V.A. \& Lazarenko E.K. (1967) Structural and mineralogical characteristics of donbassites. Mineral Sb Lvov, 21, 40-48. 
Dschang G. (1931) Die Beziehungen Zwischen Chemischer Zusammensetzung und den PhysikalischOptischen Eigenschaften in der Chloritgruppe. Doctoral Dissertation Thesis, Fischer.

Engelhardt W.V. (1942) Die Strukturen von Thuringit, Bavalit und Chamosit und ihre Stellung in der Chloritgruppe. Zeitschrift für KristallographieCrystalline Materials, 104, 142-159.

Ericsson M.T., Wäppling D.R. \& Punakivi M.K. (1977) Mössbauer spectroscopy applied to clay and related minerals. Geologiska Föreningen $i$ Stockholm Förhandlingar, 99, 229-244.

Fransolet A.M. \& Bourguignon A. (1978) Di/trioctahedral chlorite in quartz veins from the Ardennes, Belgium. The Canadian Mineralogist, 16, 365-373.

Frenzel G. \& Schembra F.W. (1965) Ein dioktaedrischer chlorit vom Kaiserbachtal (Südpfalz). Neues Jahrbuch für Mineralogie Monatshefte, 1965, 108-114.

Frondel C. (1955) Two chlorites: gonyerite and melanolite. American Mineralogist, 40, 1090-1094.

Ginzburg A.I. (1953) On lithium chlorite-cookeite. Doklady Akademii Nauk SSSR, 90, 871.

Gomes C.S.F. (1967) Alteration of spodumene and lepidolite with formation of dioctahedral chlorite plus dioctahedral chlorite-dioctahedral montmorillonite interstratifications. Publ Museu e Laboratorio Mineralogico e Geologico, Universidade de Coimbra, (Portugal), Memorias Noticias, 64, 32-57.

Goodman B.A. \& Bain D.C. (1979) Mössbauer spectra of chlorites and their decomposition products, Pp. 65-74 in: Developments in Sedimentology, Vol. 27 (M.M. Mortland and V.C. Farmer, editor). Elsevier, Amsterdam.

Gregori D.A. \& Mercader R.C. (1994) Mössbauer study of some Argentinian chlorites. Hyperfine Interactions, 83, 495-498.

Hallimond A.F., Harvey C.O. \& Bannister F.A. (1939) On the relation of chamosite and daphnite to the chlorite group. Mineralogical Magazine, 25, 441-465.

Hawes G.W. (1875) On diabantite, a chlorite occurring in the trap of the Connecticut valley. American Journal of Science, 454-457.

Hayashi H. \& Oinuma K. (1965) Relationship between infrared absorption spectra in the region of 450-900 CM-1 and chemical composition of chlorite. American Mineralogist, 50, 476.

Hödl A. (1942) Über Chlorite der Ostalpen. Ein Beitrag zur Systematik der Chlorite. Neues Jahrbuch für Mineralogie, Beil. Bd, 77, 1-77.

Holland T., Baker J. \& Powell R. (1998) Mixing properties and activity-composition and relationships of chlorites in the system $\mathrm{MgO}-\mathrm{FeO}-\mathrm{Al}_{2} \mathrm{O}_{3}-\mathrm{SiO}_{2}-\mathrm{H}_{2} \mathrm{O}$. European Journal of Mineralogy, 10, 395-406.

Holzner J. (1937) Beiträge zur kentnnis der varistischen gesteins-und mineralprovinz im Lahn-Dillgebiet. Zeitschrift für Kristallographie, Mineralogie und Petrographie, 49, 168-215.
Honda S. (1975) Dioctahedral chlorite, closely associated with kaolinite from the Kamikita mine (Kuroko deposit). Report of the Research Institute of Underground Resesarch Mining College Akita University, 43, 1-8.

Horikoshi E. (1965) Kuroko-type exhalative sedimentary mineral deposits. Journal of the Mining Institution of Kyusyu, 33, 300-210.

Hutton C.O. (1938) The Stilpnomelane Group of Minerals. Oxford University Press, Oxford, UK.

Hutton C.O. (1940) Metamorphism in the Lake Wakatipu Region (E.V. Paul, editor). Government Printer, Western Otago, New Zealand, 90 pp.

Hutton C.O. \& Seelye F.T. (1945) Contributions to the mineralogy of New Zealand - Part I. Pp. 160-168 in: Transactions of the Royal Society of New Zealand, vol. 75. (J. Hughes, editor).

Inoue A. \& Kogure T. (2016) High-angle annular dark field scanning transmission electron microscopic (HAADF-STEM) study of Fe-rich $7 \AA-14 \AA$ interstratified minerals from a hydrothermal deposit. Clay Minerals, 51, 603-613.

Inoue A., Meunier A., Patrier-Mas P., Rigault C., Beaufort D. \& Vieillard P. (2009) Application of chemical geothermometry to low-temperature trioctahedral chlorites. Clays and Clay Minerals, 57, 371-382.

Inoue A., Kurokawa K. \& Hatta T. (2010) Application of chlorite geothermometry to hydrothermal alteration in Toyoha geothermal system, southwestern Hokkaido, Japan. Resource Geology, 60, 52-70.

Joswig W. \& Fuess H. (1990) Refinement of a one-layer triclinic chlorite. Clays and Clay Minerals, 38, 216-218.

Jung H. (1931) Untersuchungen über den Chamosit von Schmiedefeld I. Thüringen. Cheme der Erde, 6, 275-306.

Jung H. \& Köhler E. (1930) Untersuchungen über den Thuringit von Schmiedefeld in Thüringen. Chemie der Erde, 5, 182.

Kawano M. \& Tomita K. (1991) Mineralogy and genesis of clays in postmagmatic alteration zones, Makurazaki volcanic area, Kagoshima prefecture, Japan. Clays and Clay Minerals, 39, 597-608.

Kimbara K. \& Nagata H. (1974) Clay minerals in the core samples of the mineralized zone of Niida, southern part of Odate Akita Prefecture, Japan. Japanese Association of Mineralogists, Petrologists and Economic Geologists Journal, 69, 239-254.

Kimbara K. \& Sudo T. (1973) Chloritic clay minerals in tuffaceous sandstone of the Miocene Green Tuff formation, Yamanaka district, Ishikawa Prefecture, Japan. Japanese Association of Mineralogists, Petrologists and Economic Geologists Journal, 68, 246-258.

Kimbara K., Shimoda S. \& Sudo T. (1973) An unusual chlorite as revealed by the high temperature X-ray diffractometer. Clay Minerals, 10, 71-78. 
Kittrick J.A. (1982) Solubility of two high-Mg and two high-Fe chlorites using multiple equilibria. Clays and Clay Minerals, 30, 167-179.

Kodama H., Longworth G. \& Townsend M.G. (1982) A Mössbauer investigation of some chlorites and their oxidation products. The Canadian Mineralogist, 20, 585-592.

Kramm U. (1980) Sudoite in low-grade metamorphic manganese rich assemblages. Neues Jahrbuch für Mineralogie Abhandlungen, 138, 1-13.

Lanari P., Wagner T. \& Vidal O. (2014a) A thermodynamic model for di-trioctahedral chlorite from experimental and natural data in the system $\mathrm{MgO}$ FeO- $\mathrm{Al}_{2} \mathrm{O}_{3}-\mathrm{SiO}_{2}-\mathrm{H}_{2} \mathrm{O}$ : applications to $\mathrm{P}-\mathrm{T}$ sections and geothermometry. Contributions to Mineralogy and Petrology, 167, 1-19.

Lanari P., Vidal O., De Andrade V., Dubacq B., Lewin E., Grosch E.G. \& Schwartz S. (2014b) XMapTools: A MATLABC-based program for electron microprobe $\mathrm{X}$-ray image processing and geothermobarometry. Computers and Geosciences, 62, 227-240.

Lapham D.M. (1958) Structural and chemical variation in chromium chlorite. American Mineralogist, 43, 921-956.

Laird J. (1988) Chlorites; metamorphic petrology. Pp. 405-453 in: Hydrous Phyllosilicates (exclusive of Micas) (S.W. Bailey, editor). Reviews in Mineralogy, 19, Mineralogical Society of America, Washington, D.C.

Lazarenko E.K. (1940) Donbassites, a new group of minerals from the Donetz Basin. CR Academy Science URSS, 28, 519-521.

Lin C.Y. \& Bailey S.W. (1985) Structural data for sudoite. Clays and Clay Minerals, 33, 410-414.

Lougear A., Grodzicki M., Bertoldi C., Trautwein A.X., Steiner K. \& Amthauer G. (2000) Mössbauer and molecular orbital study of chlorites. Physics and Chemistry of Minerals, 27, 258-269.

MacKenzie K.J.D. \& Bowden M.E. (1983) Thermal and Mössbauer studies of iron-containing hydrous silicates. IV. Amesite. Thermochimica Acta, 64, 83-106.

Malmström M., Banwart S., Lewenhagen J., Duro L. \& Bruno J. (1996) The dissolution of biotite and chlorite at $25^{\circ} \mathrm{C}$ in the near-neutral $\mathrm{pH}$ region. Journal of Contaminant Hydrology, 21, 201-213.

Malysheva T.V., Satarova L.M. \& Polyakova N.P. (1977) Thermal transformations of layered silicates and the nature of the iron-bearing phase in the CII type Murray carbonaceous chondrite. Geokhimiya, 8, 1136-1148.

Mathias M. (1952) A note on two actinolites and a chlorite from the Prieska district, Cape Province. Transactions and Proceedings of the Geological Society of South Africa, 55, 13-18.

May H.M., Acker J.G., Smyth J.R., Bricker O.P. \& Dyar M.D. (1995) Aqueous dissolution of low-iron chlorite in dilute acid solutions at 25 C. Clay Minerals Society Proceedings, Abstract, 32, p. 88.
Mélon J. (1938) Description des Chlorites et Clintonites Belges. Palais des Académies, France.

Meunier A. (2005) Clays. Springer Science \& Business Media, Berlin, $490 \mathrm{pp}$.

Mitra S. \& Bidyananda M. (2001) Crystallo-chemical characteristics of chlorites from the greenstone belt of south India, and their geothermometric significance. Clay Science, 11, 479-501.

Muñoz M., De Andrade V., Vidal O., Lewin E., Pascarelli S. \& Susini J. (2006) Redox and speciation micromapping using dispersive $\mathrm{X}$-ray absorption spectroscopy: Application to iron chlorite mineral of a metamorphic rock thin section. Geochemistry Geophysics Geosystems, 7, Doi: 10.1029/2006GC001381.

Nakamura T. (1960) On chlorite from the Ashio copper mine, Japan. Minerals Journal, 4, 383-397.

Nockholds S.R. \& Richey J.E. (1939) Replacement veins in the Mourne Mountains granites, N. Ireland. American Journal of Science, 237, 27-47.

Orcel J. (1927) Recherches sur la Composition Chimique des Chlorites. Société Générale d'Imprimerie et d'édition, France.

Pal T., Maity P.K., Das D. \& Mitra S. (1993) Oxidation character of chlorite from Byrapur chromite deposit, India - An ${ }^{57} \mathrm{Fe}$ Mössbauer evaluation. Bulletin of Materials Science, 16, 229-237.

Phillips T.L., Loveless J.K. \& Bailey S.W. (1980) $\mathrm{Cr}^{3+}$ coordination in chlorites: a structural study of ten chromian chlorites. American Mineralogist, 65, 112-122.

Poitevin E. \& Graham R.P.D. (1918) Contributions to the Mineralogy of Black Lake Area, Quebec. Government Printing Bureau, France.

Post J.L. \& Plummer C.C. (1972) The chlorite series of Flagstaff Hill area, California: A preliminary investigation. Clays and Clay Minerals, 20, 271-83.

Rigault C. (2010) Cristallochimie du fer dans les chlorites de basse température: implications pour la géothermométrie et la détermination des paléoconditions redox dans les gisements d'uranium. Thèse de doctorat, Université de Poitiers, France.

Ross C.S. (1935) Origin of the Copper Deposits of the Ducktown Type in the southern Appalachian Region. US Government Printing Office, USA.

Rule A.C. \& Bailey S.W. (1987) Refinement of the crystal structure of a monoclinic ferroan clinochlore. Clays and Clay Minerals, 35, 129-138.

Shannon E.V. \& Wherry E.T. (1922) Notes on white chlorites. Journal of the Washington Academy of Science, 12, 239.

Shimane H. \& Sudo T. (1958) A chloritic mineral found associated with vermiculite. Clay Minerals Bulletin, $\mathbf{3}$, 297-301.

Shimoda S. (1970) An expandable chlorite-like mineral from the Hanaoka Mine, Akita Prefecture, Japan. Clay Minerals Bulletin, 8, 352-359.

Shirozu H. (1958) X-ray powder patterns and cell dimensions of some chlorites in Japan, with a note 
on their interference colors. Mineralogical Journal, 2 , 209-223.

Simpson E.S. (1936) Contributions to the mineralogy of Western Australia. Royal Society of Western Australia Journal, 22, 1-18.

Simpson E.S. (1937) Contributions to the mineralogy of Western Australia. Royal Society of Western Australia Journal, 23, 17-35.

Singer D.M., Maher K. \& Brown G.E. Jr (2009) Uranylchlorite sorption/desorption: Evaluation of different U(VI) sequestration processes. Geochimica et Cosmochimica Acta, 73, 5989-6007.

Smyth J.R., Dyar M.D., May H.M., Bricker O.P. \& Acker J.G. (1997) Crystal structure refinement and Mössbauer spectroscopy of an ordered, triclinic clinochlore. Clays and Clay Minerals, 45, 544-550.

Spanu V., Filoti G., Ionescu J. \& Medesan A. (1977) Mössbauer study of some chlorites. In: Proceedings of the International Conference on Mössbauer Spectroscopy, Editorial Group of the Revue Roumaine de Physique, Bucharest, pp. 323-324.

Stone R.L. \& Weiss E.J. (1955) Examination of four coarsely crystalline chlorites by X-ray and high-pressure DTA techniques. Clay Minerals Bulletin, 2, 214-222.

Sudo T. \& Shimoda S. (1978) Clays and Clay Minerals of Japan, Vol. 26. Elsevier.

Tilley C.E. (1938) The status of hornblende in low-grade metamorphic zones of green schists. Geological Magazine, 75, 497-511.

Trincal V., Lanari P., Buatier M., Lacroix B., Charpentier D., Labaume P. \& Muñoz M. (2015) Temperature micro-mapping in oscillatory-zoned chlorite: Application to study of a greenschist facies fault zone in the Pyrenean Axial Zone (Spain). American Mineralogist, 100, 2468-2483.

Tschermak G. (1891) Die Chloritgruppe. Sitzungberichte Akademie der Wissenschaften, Wien, 100, 29 pp.

Tsukahara N. (1964) Dioctahedral chlorite from the Furutobe mine, Akita prefecture, Japan. Clay Science, 2, 56-75.

Tsuzuki Y. \& Honda S. (1977) Three examples of Mg-Al chlorite from Kuroko deposits. Journal of the Mineralogical Society of Japan, 13, 85-93.

Vidal O., Parra T. \& Trotet F. (2001) A thermodynamic model for Fe-Mg aluminous chlorite using data from phase equilibrium experiments and natural pelitic assemblages in the 100 degrees to 600 degrees C, 1 to $25 \mathrm{~kb}$ range. American Journal of Science, 301, 557-592.

Vidal O., Parra T. \& Vieillard P. (2005) Thermodynamic properties of the Tschermak solid solution in Fechlorite: Application to natural examples and possible role of oxidation. American Mineralogist, 90, $347-358$.

Vidal O., De Andrade V., Lewin E., Muñoz M., Parra T. \& Pascarelli S. (2006) P-T-deformation- $\mathrm{Fe}^{3+} / \mathrm{Fe}^{2+}$ mapping at the thin section scale and comparison with XANES mapping: application to a garnet-bearing metapelite from the Sambagawa metamorphic belt (Japan). Journal of Metamorphic Geology, 24, 669-683.

Vidal O., Lanari P., Munoz M., Bourdelle F. \& de Andrade V. (2016) Deciphering temperature, pressure and oxygen activity conditions of chlorite formation. Clay Minerals, 51, 615-633.

Vrublevskaja Z.V., Delitsin I.S., Zvyagin B.B. \& Soboleva S.V. (1975) Cookeite with a perfect regular structure, formed by bauxite alteration. American Mineralogist, 60, 1041-1046.

Weaver C.E., Wampler J.M. \& Pecuil T.E. (1967) Mössbauer analysis of iron in clay minerals. Science, 156, 504-508.

Wiewióra A. \& Weiss Z. (1990) Crystallochemical classifications of phyllosilicates based on the unified system of projection of chemical composition; II. The chlorite group. Clay Minerals, 25, 83-92.

Wilke M., Farges F., Petit P.-E., Brown G.E. \& Martin F. (2001) Oxidation state and coordination of $\mathrm{Fe}$ in minerals: An Fe K-XANES spectroscopic study. American Mineralogist, 86, 714-730.

Zazzi Å., Hirsch T.K., Leonova E., Kaikkonen A., Grins J., Annersten H. \& Edén M. (2006) Structural investigations of natural and synthetic chlorite minerals by X-ray diffraction, Mössbauer spectroscopy and solid-state nuclear magnetic resonance. Clays and Clay Minerals, 54, 252-265.

Zhang G., Burgos W.D., Senko J.M., Bishop M.E., Dong H., Boyanov M.I. \& Kemner K.M. (2011) Microbial reduction of chlorite and uranium followed by air oxidation. Chemical Geology, 283, 242-250.

Zheng H. \& Bailey S.W. (1989) Structures of intergrown triclinic and monoclinic IIb chlorites from Kenya. Clays and Clay Minerals, 37, 308-316. 